\title{
STUDYING SURFACES VIA CLOSED BRAIDS 円
}

\author{
Joan S. Birmanf \\ Department of Mathematics \\ Columbia University \\ New York, NY 10027 \\ jb@math.columbia.edu
}

\author{
Elizabeth Finkelstein[3 \\ Department of Mathematics \\ Hunter College (CUNY) \\ New York, NY 10021 \\ efinkels@shiva.hunter.cuny.edu
}

\section{$\S$ 0. INTRODUCTION.}

In the early 1980 's Daniel Bennequin wrote a seminal paper on the theory of contact structures $[\mathbf{B e}]$. The standard contact structure $\Delta$ on $\mathbb{R}^{3}$ is the kernel of the 1 -form $d z+r^{2} d \theta$, where $(r, \theta, z)$ are cylindrical coordinates in 3 -space. Thus one attaches to each point in 3 -space a planar disc whose position relative to the coordinate axes is determined by the condition $d z+$ $r^{2} d \theta=0$. The question which Bennequin addressed was whether certain other known contact structures were or were not isomorphic to $\Delta$. His beautiful insight was that this question could be referred to a question about braids and knots. Bennequin proved that if a knot was everywhere transverse to $\Delta$, then it could be deformed through transverse knots to a closed braid relative to the $z$-axis. He then proceeded to study certain incompressible spanning surfaces bounded by these closed braids, and using the foliations on those surfaces which are induced by their intersections with the halfplanes $\theta=\theta_{0}$, he found invariants of knots transverse to $\Delta$, under transverse isotopy. Using them he proved that there are contact structures which are not isomorphic to $\Delta$.

Several years after Bennequin did his work the first author of this paper and W. Menasco initiated, independently, a study of the closed braid representatives of a knot or link, using as their chief tool the combinatorics of the very same foliations on spanning surfaces as had been studied, independently, by Bennequin. In the course of their work they realized that their ideas were closely related to the ideas in $[\mathbf{B E}]$, however their interests were far from contact structures and moreover they had developed the applications to links more fully than Bennequin. They also extended the applications to the study of surfaces other than spanning surfaces for links. In addition to a series of papers which Birman and Menasco wrote (full references are

\footnotetext{
${ }^{1}$ To appear in J. of Knot Theory and its Ramifications, Volume 7, No. 3 (1998)

${ }^{2}$ partially supported by NSF Grants DMS 91-06584 and 94-02988

${ }^{3}$ partially supported by a summer grant from Dartmouth College
} 
given below) their techniques were exploited in other directions and used in diverse ways by J. Los, E. Finkelstein, P. Cromwell and T.S. Fung. In particular, the machinery was applied to the following types of surfaces:

1. An incompressible spanning surface for a link $L$ (see [Be, BM 1, [BM 2], [BM 3], [BM 5], [BM 6], [KL], [L]).

2. A closed incompressible surface in the complement of $L$ (see BM 4, [BM 7, , Fin, C]).

3. An incompressible 2 -sphere in the complement of $L$ which divides $L$ into irreducible components BM 4

4. A closed 2-sphere which decomposes a non-prime component $K$ of $L$ into prime summands (see [BM 4]).

5. A family of immersed 'unknotting' discs which $L$ bounds $\mathbb{M}$

6. An immersed ribbon surface which $L$ bounds [Fu].

While all of the papers cited above used related techniques, those techniques were developed in an ad hoc fashion, to fit the needs of the moment. It was only after they had been used repeatedly that it was realized that a general reference might be in order. That is the purpose of this paper: to review the Bennequin-Birman-Menasco machinery, in a systematic fashion, in the most general setting which we know at this time, with an eye toward making it accessible to the beginning reader. It is our intention that this paper will serve as a basic reference for the manuscripts [Fin] and [Fu]. We will also give several new applications and state some open research problems. The potential applications do not appear to have been exhausted.

Here is a guide to the paper. In Section 1 we set up our basic geometry. The main result in Section 1 is that incompressible spanning surfaces for links and closed incompressible surfaces in link complements admit a singular foliation which allows them to be divided into a finite number of foliated regions, each of which has a canonical embedding in 3-space. See Theorem 1.2. In Section 2 we describe ways that we can modify the foliation locally via isotopy, and in some cases this involves a change in the braid representation. In Section 3 we describe certain global properties of the foliation. In Section 4 we give examples of ways in which the machinery of Sections 1, 2 and 3 can be applied, and we pose several interesting open problems.

The new results in this paper may be summarized as follows:

- The 'change in foliation' move of $\S 2.1$ is stated and proved in a new way, which clarifies its content. 
- The 'exchange moves' of $\S 2.4$ have been described in a new way which clarifies the fundamental way in which these moves depend on the surface foliation.

- The graphs which are studied in $\S 3$ are investigated here for the first time as a systematic tool. Theorem 3.1, which shows how the graphs can be simplified by changes in foliation, exchange moves, isotopies and deletions of trivial loops, is a new result.

- The proof of Theorem 4.3, Markov's Theorem without stabilization, in the special case of the unlink, is new. This concise proof is possible due to the use of the graphs of $\S 3$.

- Example 4.1 is studied here in a systematic way for the first time. It illustrates that the foliation of a surface bounded by a knot can indicate how different representatives of the knot are related.

Acknowledgement: We thank the referee for his or her careful reading of the manuscript and many constructive suggestions. We thank Bill Menasco for numerous helpful conversations. Many of the results in this paper are based upon his work, and his joint work with the first author. We thank Michael Hirsch for pointing out an oversight in the statement and proof of Theorem 2.1. We thank Tahl Nowik for pointing out a missing case in the proof of Lemma 1.4 and for showing us that the proof follows from his work in $[\mathbb{N}]$.

\section{$\S$ 1. BASIC MACHINERY.}

The central idea in the study of surfaces via closed braids is that when a link is positioned as a closed braid in $S^{3}$, there is a foliation of the complement of the braid axis by discs whose boundary is the axis. This foliation induces (by intersection) a singular foliation of any surface whose position is related to the position of a given link. The purpose of this section is to describe the general position arguments which we use to make this foliation amenable to study, and then to begin to study it.

We restrict our attention throughout this paper to surfaces which are embedded in non-split link complements $S^{3} \backslash L$. We use: 
- the symbol $\mathcal{F}$ for an incompressible spanning surface for $L$, oriented so that the positive normal bundle to each component has the orientation induced by that on $L=\partial \mathcal{F}$.

- the symbol $\mathcal{C}$ for a closed oriented incompressible surface in $S^{3} \backslash L$, and

- the symbol $\mathcal{S}$ when our work refers to both $\mathcal{F}$ and $\mathcal{C}$.

We will not consider the case of a 2 -sphere which pierces a single component of $L$ twice, because that case is rather special, moreover it follows easily from the others. We will not consider split links, but many of the results hold in this case as well (see, for example, [BM 4). We refer the reader who is interested in immersed surfaces to the manuscript of T.S. Fung [Fu]. That manuscript includes a discussion of what is known, at this writing, about the applications of the machinery which is developed in this paper to the study of immersions.

A link type $\mathcal{L}$ has a closed $n$-braid representative $L$ if there is an unknot $A$ in $S^{3} \backslash L$, and a choice of fibration $H$ of the solid torus $S^{3} \backslash A$ by meridian disks, such that $L$ intersects each fiber of $H$ transversely. Sometimes it will be convenient to replace $S^{3}$ by $R^{3}$ and to think of the fibration $H$ as being by half-planes $\left\{H_{\theta} ; \theta \in[0,2 \pi]\right\}$ of constant polar angle $\theta$ through the $z$-axis. Note that $L$ intersects each fiber $H_{\theta}$ in the same number of points, that number being the braid index $n$ of $L$. We may always assume that $L$ and $A$ can be oriented so that $L$ travels around $A$ in the positive direction, using the right hand rule, as in Figure 1.1.

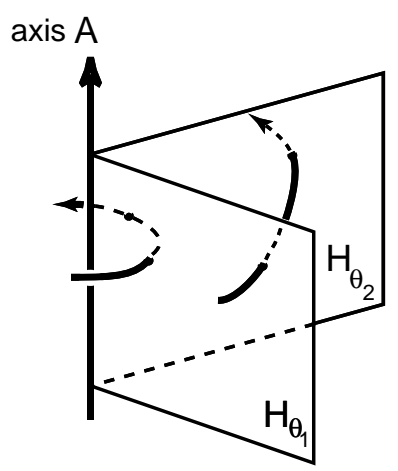

Figure 1.1

It was proved by Alexander [A] that every link type may be represented as a closed $n$-braid, for some $n$. 
The braid axis and the fibers of $H$ will be seen to serve as a coordinate system in 3-space in which to study closed surfaces in $R^{3} \backslash L$ and spanning surfaces for the link. A singular foliation of each such surface is induced by its intersection with fibers of $H$. We show (see Theorem 1.2) that the surface is decomposed into a finite set of canonical regions, each of which is embedded in 3-space (relative to our coordinates) in a canonical way. Later (see Theorem 4.1) we will show that the surface can be described via a finite set of combinatorial data associated to this foliation. The combinatorial data determines the surface embeddings by describing how the canonical pieces fit together.

Our initial goal in this section is to use some very simple and well-known general position techniques to place restrictions on the foliation of $\mathcal{S}$. After that we will prove several basic lemmas which allow us to restrict the leaf type and to assume that the foliation has no 'inessential' leaves. Our first main result is Theorem 1.1.

By general position, we may assume that:

(i) The intersections of $A$ and $\mathcal{S}$ are finite in number and transverse.

(ii) There is a neighborhood $N_{A}$ of $A$ in $R^{3} \backslash L$ such that each component of $\mathcal{S} \cap \mathcal{N}_{\mathcal{A}}$ is a disk, and each disk is radially foliated by its arcs of intersection with fibers of $H$.

(iii) There is a neighborhood $N_{L}$ of $L$ in $R^{3}$ such that $N_{L} \cap \mathcal{F}$ is foliated by arcs of intersection with fibers of $H$ which are transverse to $L$.

(iv) All but finitely many fibers $H_{\theta}$ of $H$ meet $\mathcal{S}$ transversely, and those which do not (the singular fibers) are each tangent to $\mathcal{S}$ at exactly one point in the interior of both $\mathcal{S}$ and $H_{\theta}$. Moreover, each point of tangency is a local maximum or minimum or a saddle point (with respect to the parameter $\theta$ ).

A singular leaf in the foliation is one which contains a point of tangency with a fiber of $H$. All other leaves are non-singular. It follows from (iv) that:

(v) Each non-singular leaf is an arc or a simple closed curve.

(vi) Each singular fiber contains exactly one singularity of the foliation, each of which is a center or a saddle point.

Note that each non-singular leaf in the foliation of $\mathcal{C}$ which is an arc must have both endpoints on $A$, since $\mathcal{C}$ does not intersect $L$. On the other hand, 
arcs in the foliation of $\mathcal{F}$ may, in principle, have endpoints on either $A$ or $L$. The following lemma, however, rules out the case of an arc which has both endpoints on $L$.

Lemma 1.1. If $H_{\theta}$ is a non-singular fiber of $H$, then an arc in $H_{\theta} \cap \mathcal{F}$ cannot have both of its endpoints on $L$.

Proof of Lemma 1.1. Let $\alpha \in H_{\theta} \cap \mathcal{F}$ be an arc which has both of its endpoints on L and let $N_{\alpha}$ be a neighborhood of $\alpha$ on $\mathcal{F}$ (see Figure 1.2). Then $L \cap N_{\alpha}$ has two components, $k$ and $k^{\prime}$, which have opposite orientations as subarcs in the boundary of the oriented surface $N_{\alpha}$. However, $L$ is a closed n-braid, hence it meets each $H_{\theta}$ in $n$ coherently oriented transverse intersections. Since $\alpha$ lies in both $\mathcal{F}$ and $H_{\theta}$, and since $N_{\alpha}$ intersects $H_{\theta}$ transversely, this is impossible. $\|$

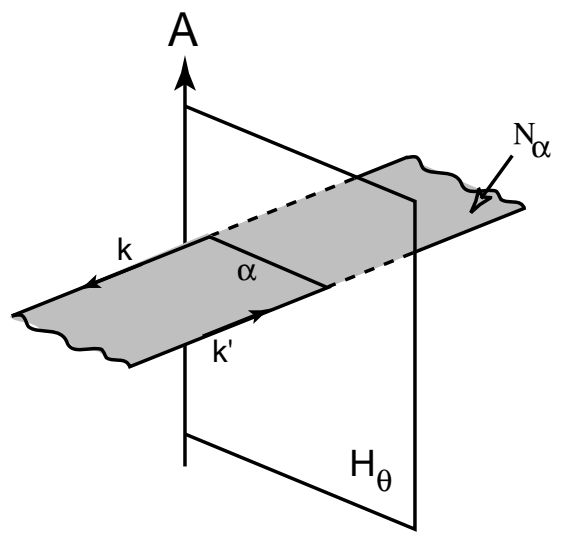

Figure 1.2

In view of Lemma 1.1, the non-singular leaves in the foliation of $\mathcal{S}$ can be subdivided into three types:

$a$-arcs: arcs which have one endpoint on $A$ and one on $L=\partial \mathcal{S}$

b-arcs: arcs which have both endpoints on $A$.

c-circles: simple closed curves

Each $b$-arc in a fiber $H_{\theta}$ separates that fiber into two components. Call the $b$-arc essential if each of these components is pierced at least once by $L$, and inessential otherwise. Each $c$-circle in a fiber $H_{\theta}$ bounds a disk $D_{c}$ in $H_{\theta}$. The $c$-circle is essential if $D_{c}$ is pierced at least once by $L$. It is inessential otherwise. We say the $c$-circle is a meridian, if $D_{c}$ intersects $L$ in exactly one point (note that the interior of $D_{c}$ may intersect the surface). 
Lemma 1.2. All b-arcs in the foliation of $\mathcal{S}$ may be assumed to be essential.

Proof of Lemma 1.2. If the foliation of $\mathcal{S}$ contains an inessential $b$-arc, that arc will cobound with a segment of the axis $A$ a disk $\Delta$ in some $H_{\theta}$ (see Figure 1.3). We can then push the surface in along a neighborhood of $\Delta$ in $R^{3}$ to remove two points of intersection of the surface with the axis $A$. The removal of the inessential $b$-arc reduces the number of points at which $A$ intersects $\mathcal{S}$. Since the number of points in $A \cap \mathcal{S}$ is finite, we can continue this process until all inessential $b$-arcs have been removed. $\|$

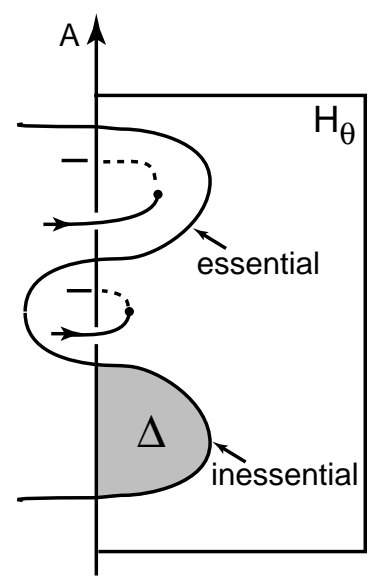

Figure 1.3

Lemma 1.3. If the foliation of $\mathcal{F}$ contains c-circles, then $\mathcal{F}$ is isotopic to a spanning surface $\mathcal{F}^{\prime}$ for $L$ which is foliated without c-circles.

Proof of Lemma 1.3. Suppose that $c$ is a $c$-circle in the foliation of an incompressible spanning surface $\mathcal{F}$ for $L$. Moving through the fibration slightly, we may assume that $c$ is contained in a non-singular fiber $H_{c}$. Let $D_{c}$ be the disk in $H_{c}$ bounded by $c$. If $L$ intersects $D_{c}$, then there must be arcs in the foliation of $\mathcal{F}$ of the type ruled out by Lemma 1.1. Therefore we can assume all $c$-circles in the foliation of $\mathcal{F}$ are inessential.

Let $c$ be an inessential $c$-circle in the foliation of $\mathcal{F}$ contained in a nonsingular fiber $H_{c}$. The entire surface cannot be foliated by inessential circles, since then it would be a compressible torus. Therefore if we follow $c$ as it evolves in the fibration, we must arrive in one direction at a circle $c_{\theta}$ containing a singular point $p_{\theta}$ in a singular fiber $H_{\theta}$. The point $p_{\theta}$ is either a center or a saddle point, but if it is a center we can reverse the flow and find a saddle. So, we may assume it is a saddle. By property (iv) $p_{\theta}$ is the only singularity on the fiber $H_{\theta}$, so the singularity must be as shown in Figure 
1.4. There are two possibilities, illustrated in Figure 1.4, but if the second occurs then the first occurs for some other choice of inessential $c$, so we may assume we are in the situation of case 1 .

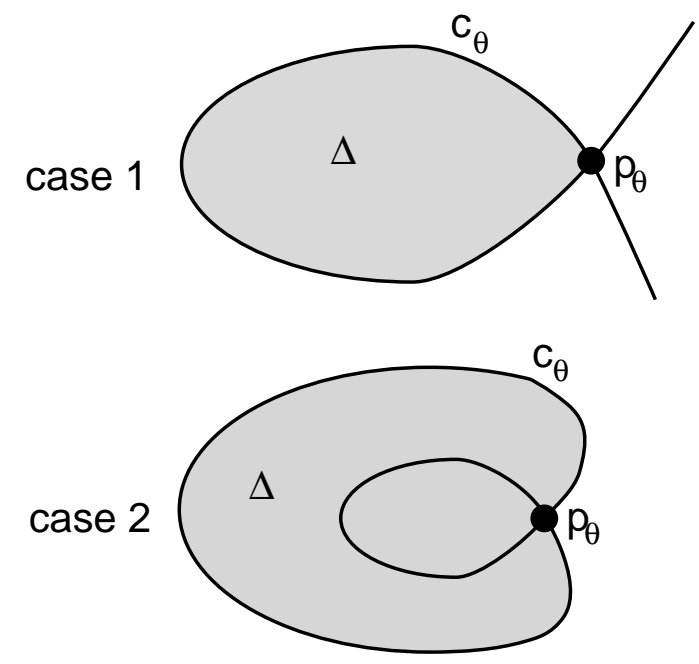

Figure 1.4

Thus $c_{\theta}$ bounds a disc $\Delta$ in $H_{\theta}$ as shown in case 1 , and $\Delta$ does not intersect $L$ since all $c$-circles in the foliation of $\mathcal{F}$ are inessential. It may happen that the interior of the disk $\Delta$ has empty intersection with $\mathcal{F}$. If so, we may surger $\mathcal{F}$ along $c_{\theta}$ as in Figure 1.5 (a). Since $\mathcal{F}$ is incompressible, the surface resulting from this surgery must have one more component than $\operatorname{did} \mathcal{F}$, and one of the new components must be a topological 2-sphere. Discarding the 2-sphere, we obtain a new incompressible spanning surface for $L$. A small isotopy smooths the surface out so that the saddle $p_{\theta}$ and the circle $c_{\theta}$ disappear, and the fibration of the resulting surface has at least one less saddle singularity than that of the original surface $\mathcal{F}$.

It remains to consider the case in which the interior of $\Delta$ intersects $\mathcal{F}$. By property $(i v)$, there are no singularities of $\mathcal{F} \cap \mathcal{H}_{\theta}$ in the interior of $\Delta$, so each component of $\mathcal{F} \cap\rangle \backslash \sqcup(\cdot)$ must be a $c$-circle. Let $c^{\prime}$ be an innermost such $c$-circle bounding a disk $D$ in $H_{\theta}$. Since $c$ is inessential, we may surger $\mathcal{F}$ along $D$, as in Figure 1.5 (b). As before, the surgery produces a closed component $X$ homeomorphic to $S^{2}$. This 2-sphere must be tangent to $H$ at at least two points, so discarding $X$ eliminates at least two centers. Thus, although the surgery itself introduces two new centers, discarding $X$ results in a surface with no more centers or saddles than the original. A finite number of surgeries of this type result in the disk $\Delta$ having interior disjoint from $\mathcal{F}$. A surgery of the type shown in Figure 1.5 (a) then eliminates it 
along with its associated saddle singularity.

Note that the existence of a $c$-circle implies the existence of a saddle singularity and that, in each case, the surgeries which eliminate it result in a reduction in the total number of saddle singularities. Induction on the number of saddle singularities then allows us to conclude that, after finitely many surgeries, a surface $\mathcal{F}^{\prime}$ is obtained which is foliated without $c$-circles. Our final observation is that, since the link $L$ is non-split, each time we do a surgery the 2-sphere $X$ must bound a 3 -ball in $S^{3} \backslash L$. It follows that each surgery could have been replaced by an isotopy of the surface through a 3 -ball. Thus, the resulting surface $\mathcal{F}^{\prime}$ is isotopic to $\mathcal{F}$. $\|$

Lemma 1.4. If the foliation of $\mathcal{C}$ contains inessential c-circles, then $\mathcal{C}$ is isotopic to a surface $\mathcal{C}^{\prime}$ in $S^{3} \backslash L$ which is foliated without inessential c-circles.

Proof of Lemma 1.4. The argument which we used to eliminate inessential $c$-circles in the proof of Lemma 1.3 applies as before, except possibly in the situation of case 2 of Figure 1.4. In case 2, when the surface is closed and has genus greater than zero and the $c$-circle "surgers with itself from the outside," it can happen that the disk which is bounded by the inner circle after the surgery has non-empty intersection with the link $L$. We are grateful to Tahl Nowik for pointing this out to us, and for showing us how this lemma follows from his work in $[\mathbf{N}]$.

In $\mathbb{N}$ Nowik studies the intersection of closed, orientable incompressible surfaces $S$ and $F$ in an irreducible orientable 3-manifold $M$. The surface $S$ is fixed while $F$ moves via a directed isotopy toward a preferred side. Throughout the isotopy $F$ is in general position relative to $S$. Now let $M$ be the closure of $S^{3} \backslash N$, where $N=N_{A} \cup N_{L}, N_{A}$ is a neighborhood of the axis $A$ and $N_{L}$ is a neighborhood of the link $L$. Let $F$ be the closure of $H_{\theta} \backslash\left(N \cap H_{\theta}\right)$ and let $S$ be the closure of $\mathcal{C} \backslash(\mathcal{N} \cap \mathcal{C})$. Except for the fact that these surfaces $S$ and $F$ have non-empty boundary, this is exactly the situation studied in $[\mathbf{N}]$. Our situation is quite restricted, however, as $\partial F$ consists of meridians of $\partial N_{L}$ and a longitude of $\partial N_{A}$, and $\partial S$ consists of meridians of $\partial N_{A}$. Therefore, since each meridian of $\partial N_{A}$ has a neigborhood on $S$ foliated entirely by arcs of intersection with fibers of $H$, all singularities between $F$ and $S$ which occur as $F$ moves through the fibration are off $\partial F$ and $\partial S$. The reader can verify that the proof of Theorem 6.1 of [N] holds in this special case, and that this lemma follows as an immediate consequence. $\|$ 

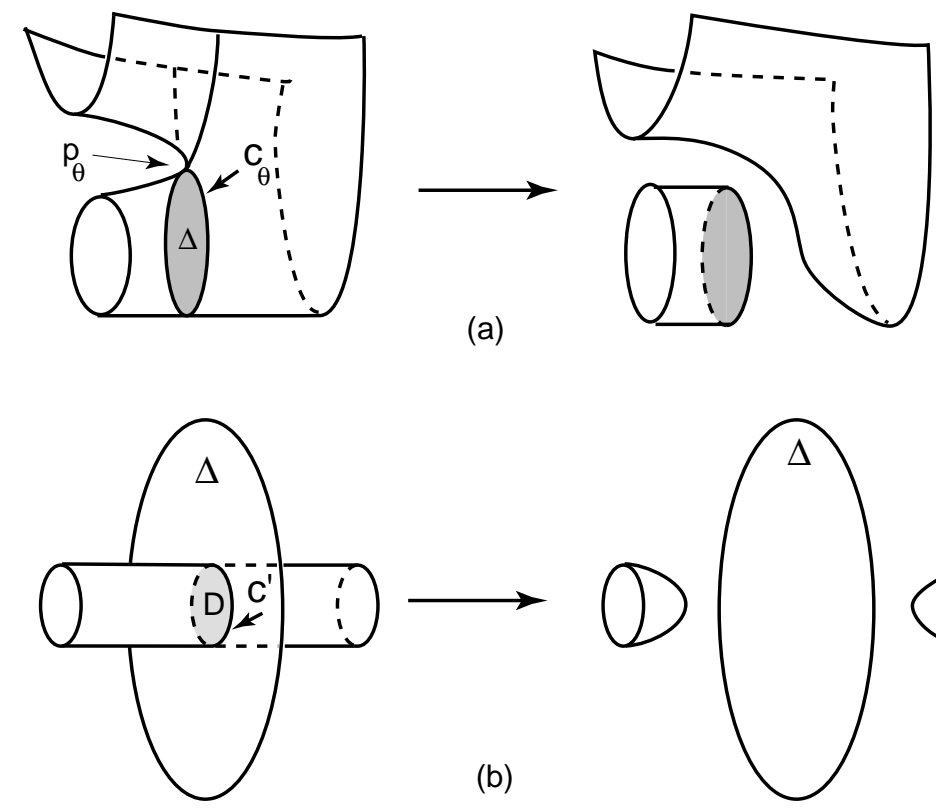

(1)

Surgery followed by isotopy

Figure 1.5

Lemma 1.5. All singularities in the foliation of $\mathcal{S}$ may be assumed to be saddles.

Proof of Lemma 1.5. Suppose there is a center. Proceeding through the fibration, we obtain a $c$-circle in the fiber $H_{c}$ bounding a disk $D$ contained in $\mathcal{S}$ (see Figure 1.6). The circle $c$ is essential, so it bounds a disk $D_{c} \subset H_{c}$ intersecting $L$ algebraically non-zero times. Gluing $D$ to $D_{c}$ along $c$ results in a 2 -sphere pierced algebraically non-zero times by $L$. Impossible $\|$

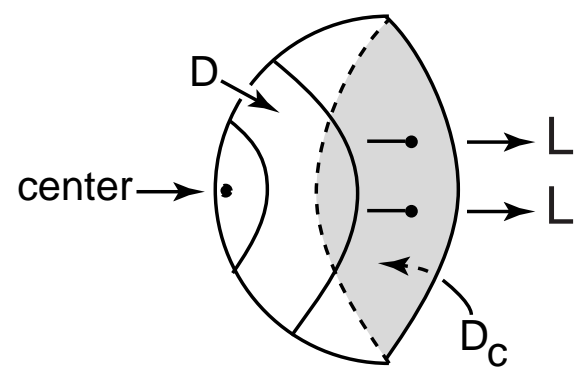

Figure 1.6 
Note that, moving forward through the fibration, any singular leaf in the foliation is formed by non-singular leaves moving together to touch at a saddle singularity. After the saddle, the singular leaf is transformed into new non-singular leaves. We call this process a surgery of the non-singular leaves, and label the singularities, singular leaves and corresponding surgeries according to the non-singular leaves associated to them (see Figure 1.7):

type aa: an $a$-arc surgered with an $a$-arc, type $a b$ : an $a$-arc surgered with a $b$-arc, type $b b$ : a $b$-arc surgered with a $b$-arc, type $b c$ : a $b$-arc surgered with itself or a $c$-circle, type $c c$ : a $c$-circle surgered with itself or another $c$-circle.

The preceding lemmas, together with the fact that the foliation of a closed surface contains no $a$-arcs, imply the following theorem.

\section{Theorem 1.1}

(i) The surface $\mathcal{F}$ can be chosen so that every non-singular leaf in its foliation is an a-arc or an essential b-arc, and every singularity in its foliation has type $a a, a b$ or $b b$.

(ii) The surface $\mathcal{C}$ can be chosen so that every non-singular leaf in its foliation is an essential b-arc or an essential c-circle, and every singularity in its foliation has type bb, bc or cc.

From now on, we assume that $\mathcal{S}$ has been chosen so that its foliation is as described in Theorem 1.1.

We next show how the foliation of $\mathcal{S}$ allows us to decompose $\mathcal{S}$ into very simple canonical pieces, which have canonical embeddings in 3-space. Our results will be summarized, later, in Theorem 1.2.

Note that no component $\mathcal{X}$ of $\mathcal{S}$ is foliated entirely by $b$-arcs, since then $\mathcal{X}$ is a 2 -sphere, contradicting the incompressibility of $\mathcal{S}$. Thus, if the foliation of $\mathcal{X}$ contains no singularities, $\mathcal{X}$ is either a disk foliated by $a$-arcs (in the case $\mathcal{S}=\mathcal{F}$ ) or a torus foliated by $c$-circles (in the case $\mathcal{S}=\mathcal{C}$ ). Otherwise, let $U$ be the union of all the singular leaves in the foliation of $\mathcal{X}$. Since the fibration $H$ has finitely many singular fibers, each singular leaf $\lambda$ in $U$ has a 

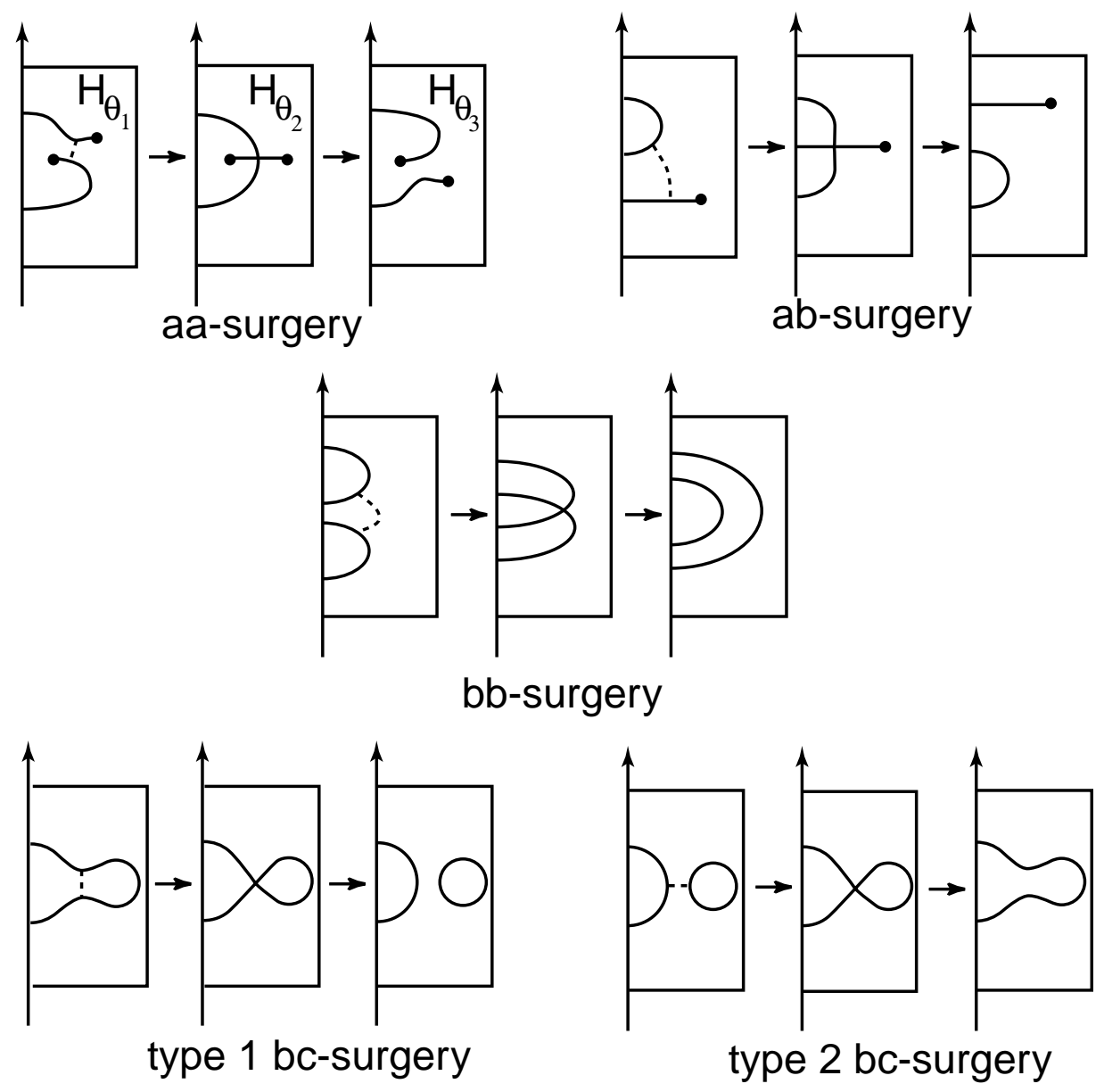

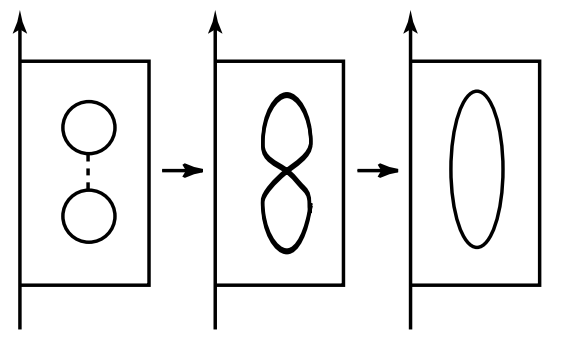

type 1 cc-surgery

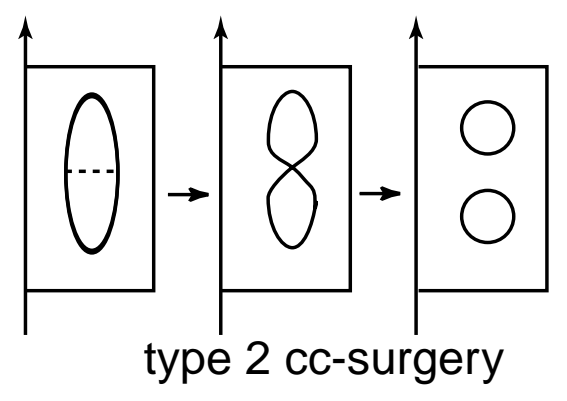

Figure 1.7 
foliated neighborhood $N_{\lambda}$ in $\mathcal{S}$ such that $N_{\lambda} \cap U=\lambda$. If $\lambda$ has type $a a, a b$, or $b b, N_{\lambda}$ is one of the foliated open 2-cells shown in Figure 1.8, with the arrows indicating the direction of increasing $\theta$.
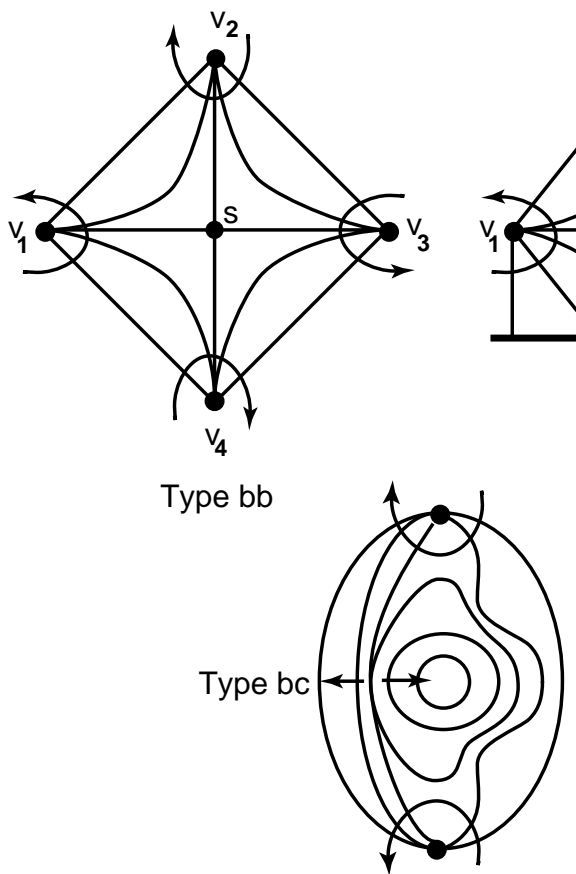

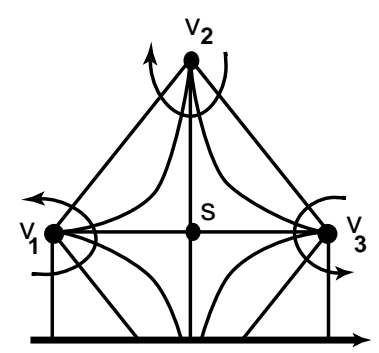

Type ab

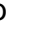

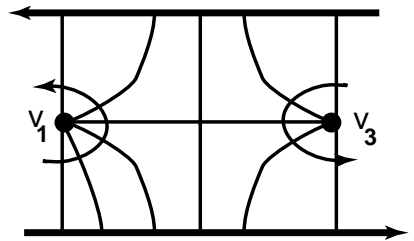

Type aa

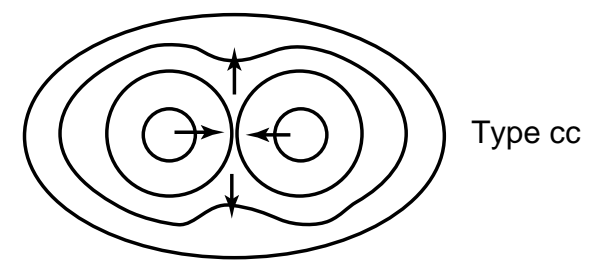

Figure 1.8

If $\lambda$ has type $b c$ or $c c, N_{\lambda}$ is a foliated annulus or twice-punctured disk, respectively. Figure 1.8 shows this in the case of the type $1 b c$ and $c c$ surgeries described in Figure 1.7. The foliated neighborhoods for the case of type 2 surgeries are obtained by reversing the arrows.

The complement of $U$ in $\mathcal{X}$ is a union $B_{1} \cup B_{2} \cup \ldots \cup B_{k}$, where each $B_{i}$ is foliated entirely by non-singular leaves as shown in Figure 1.9. If we choose one non-singular leaf in each $B_{i}$ and declare it to be a boundary arc or boundary circle, then the union of all the boundary arcs and circles determines a decomposition of $\mathcal{X}$ into regions, each of which is a foliated neighborhood of one singular leaf. Let the type of a boundary arc be type a or type $b$ according to whether it is an $a$-arc or $b$-arc, respectively. 

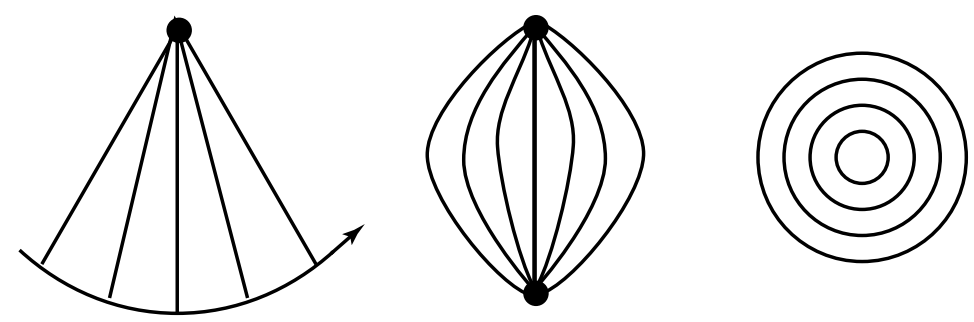

Figure 1.9

From Theorem 1.1, we know that the decomposition of $\mathcal{F}$ contains only regions of type $a a, a b$, and $b b$. Since these are 2-cells, the decomposition of $\mathcal{F}$ is a foliated cell-decomposition. We will refer to these as tiles of type $a a$, $a b$ and $b b$, and to the cell-decomposition a tiling.

Remark 1.1 Observe from Figure 1.8 that an $a b$-tile can be obtained from a $b b$-tile by replacing one vertex of the $b b$-tile with a subarc of the link. Similarly, an $a a$-tile can be obtained from an $a b$-tile by replacing one vertex of the $a b$-tile with a subarc of the link.

Singularities have a sign. We assume that the fibers $H_{\theta}$ are positioned so that the direction of the positive normal to $H_{\theta}$ agrees at each point with the direction of increasing $\theta$. At a singular point $s$ in the foliation the normal to $\mathcal{S}$ at $s$ coincides with the normal to $H_{\theta}$ at $s$. We say that the singularity at $s$ is positive (or negative) according as the outward-drawn normal to $\mathcal{S}$ is pointing in the direction of increasing (or decreasing) $\theta$. Figure 1.10 shows a positive singularity viewed in a sequence of fibers of $H$. Let the type (respectively sign) of a region be equal to the type (respectively sign) of the singularity it contains.

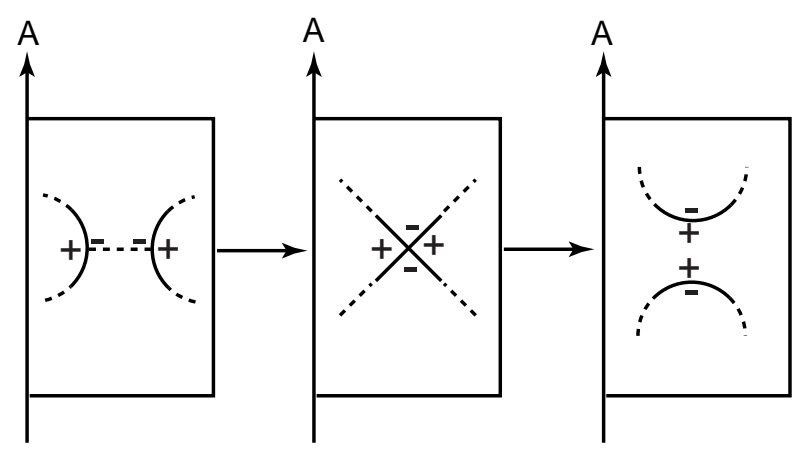

Figure 1.10 
If the foliation of a surface contains $a$-arcs or $b$-arcs, then the axis $A$ intersects the surface in a finite number of points. Call these points vertices of the tiles. Each vertex $v$ is an endpoint of finitely many boundary arcs in the surface decomposition. Let the type of $v$ be the cyclic sequence $\left(x_{1}, \ldots, x_{r}\right)$, where each $x_{i}$ is either $a$ or $b$, and the sequence lists the types of region boundary arcs meeting at $v$ in the cyclic order in which they occur in the fibration. The valence of $v$ is the number of distinct regions in the surface decomposition intersecting at $v$. The sign of $v$ is the cyclic array of signs of the regions meeting at $v$. We define the parity of $v$ to be positive or negative according as the outward-drawn normal to the surface has the same or opposite orientation as the braid axis at the vertex. This means that when we view the positive side of the surface, the sense of increasing $\theta$ around a vertex will be counterclockwise (resp. clockwise) when the vertex is positive (resp. negative), as illustrated in Figure 1.11.
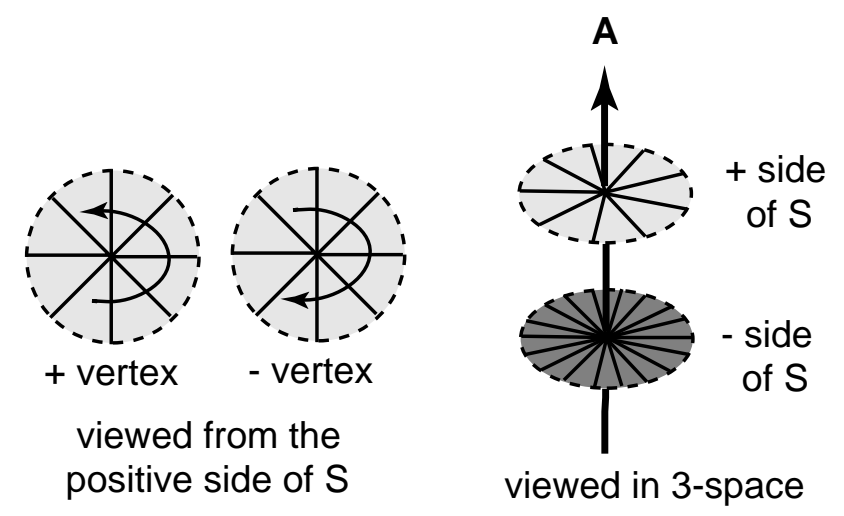

Figure 1.11

Lemma 1.6 Regions of type $a a, a b, b b$ each have a canonical embedding in 3 -space, which is determined up to an isotopy of 3-space which preserves the axis $A$ and each fiber of $H$ setwise and up to the choice of the sign of the singularity. Regions of type $b c, c c$ each have two such canonical embeddings, which are the same up to a choice of direction of increasing $\theta$.

Proof of Lemma 1.6. We illustrate the proof by showing how to embed a tile $T$ of type $a b$ in 3-space. Initially, it will be most convenient to work in $S^{3}$, so let us think of the axis $A$ as a circle and the fibers of $H$ as discs. Choose an $a b$-tile with vertices at $v_{1}, v_{2}, v_{3}$, as in Figure 1.12(a). Let $s$ be the singular point in the foliation of $T$ and let $p$ be the $L$-endpoint of the singular leaf in $T$. 


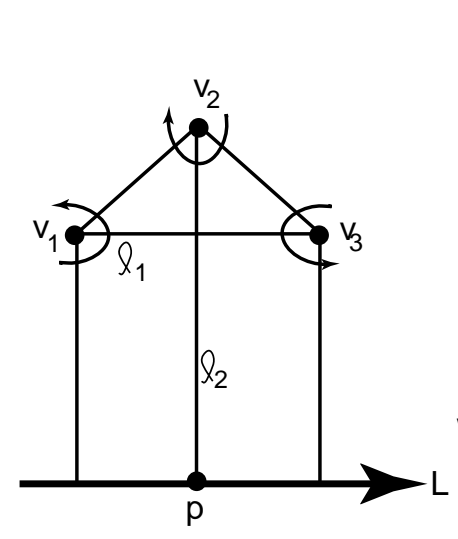

(a)

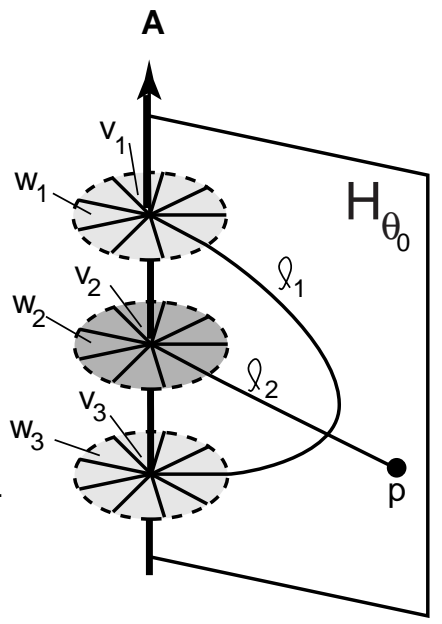

(b)

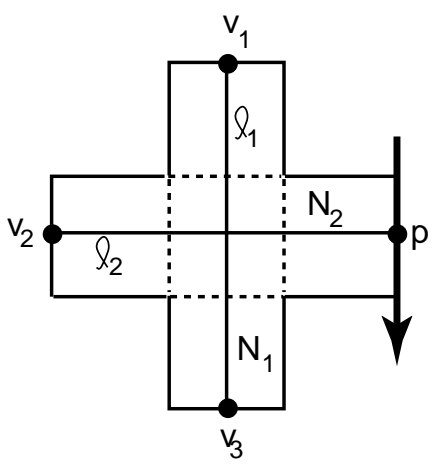

(c)

Figure 1.12

The vertices are on $A \cap \mathcal{S}$, where they have a natural cyclic order, i.e either $v_{1}, v_{2}, v_{3}$ or its reverse. One arc of the singular leaf contained in $T$, say $l_{1}$, runs from $v_{1}$ to $v_{3}$, separating $H_{\theta_{0}}$ into 2 halves, one of which contains $v_{2}$. The other half of $H_{\theta_{0}}$ must contain $p$, because the other arc of the singular leaf $l_{2}$, which runs from $v_{2}$ to $p$, crosses the singular leaf $l_{1}$. The point of intersection $l_{1} \cap l_{2}$ is $s$.

We now pass to $R^{3}$, choosing the point at infinity so that it separates $v_{1}$ and $v_{3}$. Our fiber $H_{\theta_{0}}$ is now a half-plane through the axis, containing the singular leaf $l_{1} \cup l_{2}$ which we just constructed. We wish to embed the rest of the tile $T$ in 3 -space. Since the only singularity on $T$ is the one at $s$, the rest of $T$ will be transverse to the fibers it intersects. The first step is to color the two sides of $T$ light grey (for positive) and dark grey (for negative). Let $w_{i}$ be a radially foliated neighborhood of $v_{i}$ on $T$, i.e. $w_{i}$ is a sector of a disc. We embed each $w_{i}$ in 3 -space so that it is orthogonal to $A$ and attached to $A$ at $v_{i}$. Since $v_{1}$ and $v_{3}$ are positive and $v_{2}$ is negative, the light grey sides of $w_{1}$ and $w_{3}$ face up and the dark grey side of $w_{2}$ faces up (see Figure 1.12(b)).

Next choose two overlapping rectangular strips which are neighborhoods $N_{1}$ and $N_{2}$ of the $\operatorname{arcs} l_{1}$ and $l_{2}$ on $T$ (see Figure $1.12(\mathrm{c})$ ) and paste them to the $w_{i}^{\prime} s$ and the singular leaf so that they are transverse to the fibers of $H$ everywhere except along the singular leaf. Since the light grey sides of both $w_{1}$ and $w_{3}$ are facing up, we will need to give the strip $N_{1}$ a half-twist as we attach it. (We cannot give it additional twists because its long edges must be transverse to the fibers of $H$.) The half-twist has a sign, determined by 
the sign of $s$ : the light grey (or dark grey) side of $N_{1}$ faces in the direction of increasing $\theta$ according as $s$ is positive (or negative). We now pass to $N_{2}$. The dark grey side of $w_{2}$ faces up, so there is a unique way to paste $N_{2}$ to $w_{2}$. The other end of $N_{2}$ is glued to $L$, and we know how to glue it because $L$ is oriented and $S$ has the induced orientation. We also know that $N_{2}$ is twisted just enough so that it can be glued to $N_{1}$ along the disc $N_{1} \cap N_{2}$ and so that its long edges are transverse to the fibers of $H$. The full picture of an embedded $a b$-tile is shown in the top row of Figure 1.13.

The type $a a, b b$ cases are similar, and the reader will have no difficulty in constructing the embeddings shown in Figure 1.13, with the help of the pictures given earlier in Figure 1.8. The type 1 and type 2 embeddings for each of the $b c$ and $c c$ regions may also be constructed in a similar fashion, using the type 1 and type 2 foliations shown in Figure 1.8, respectively. Note that, in each case, the type 1 and type 2 embeddings in $S^{3}$ are identical, if the direction of increasing $\theta$ and the orientation of $\mathcal{S}$ are reversed. This follows from the fact that the corresponding type 1 and type 2 surgeries are identical, but time-reversed (see Figure 1.7). ॥

Remark 1.2 As we mentioned in Remark 1.1, an $a b$-tile can be obtained from a $b b$-tile and an $a a$-tile can be obtained from an $a b$-tile, by replacing one vertex with a subarc of the link. The reader can check that the embeddings of the $a b$-tile and $a a$-tile in Figure 1.13 can be obtained in this manner for a suitable choice of embedding for the subarc of the link. We have chosen these particular embeddings to make the singularities of the foliation visible. We have also distorted the embedding of the $a a$-tile slightly in order to make its singularity more visible.

Summarizing, we have proved:

\section{Theorem 1.2}

(1.2.1) Each component of $\mathcal{F}$ is a disk foliated by a-arcs or is decomposed into canonically embedded regions of type $a a, a b, b b$, each of which contains exactly one singular point in the foliation.

(1.2.2) Each component of $\mathcal{C}$ is a torus foliated by c-circles or is decomposed into canonically embedded regions of type $b c, c c$, each of which contains exactly one singular point in the foliation. 

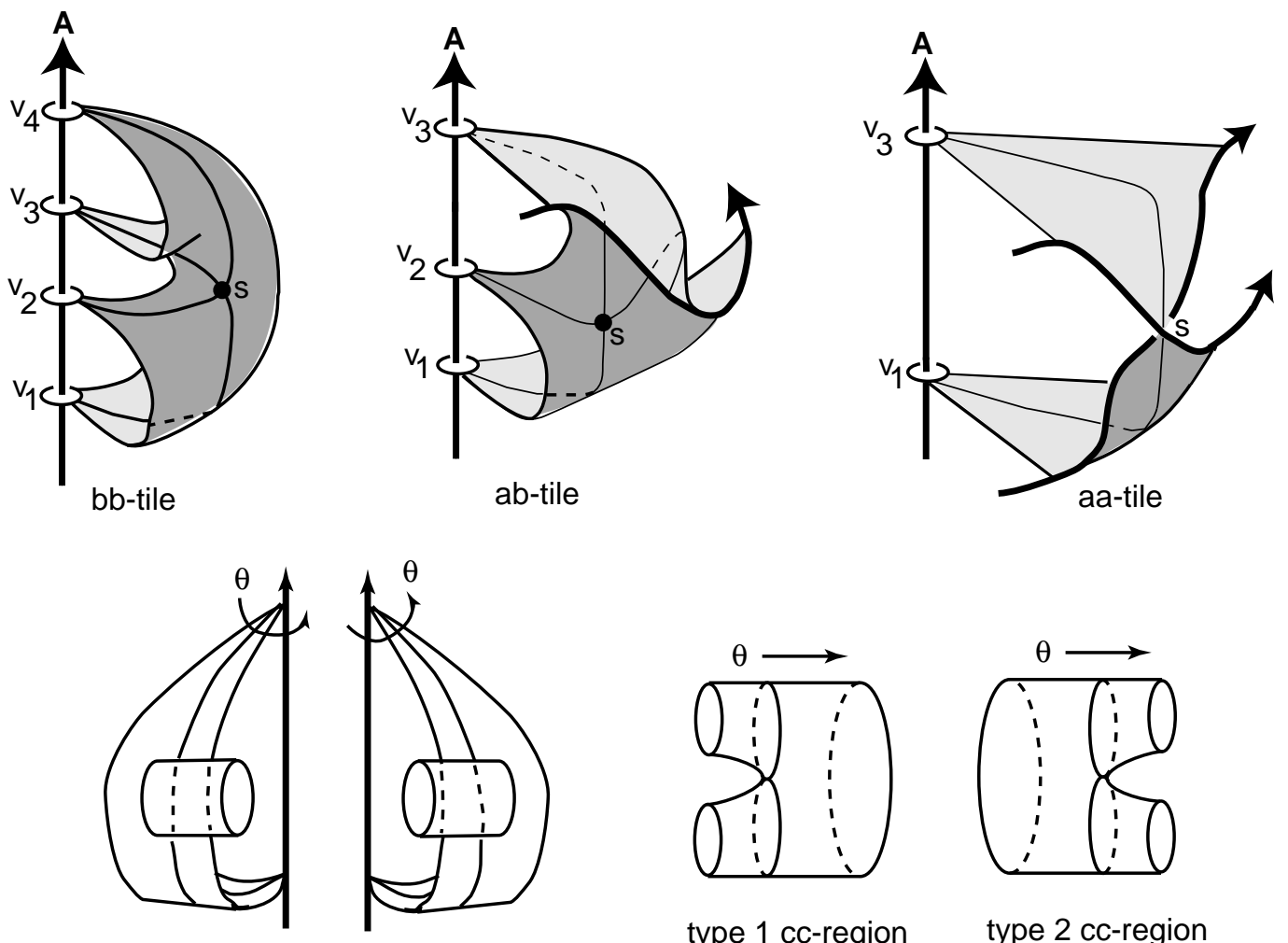

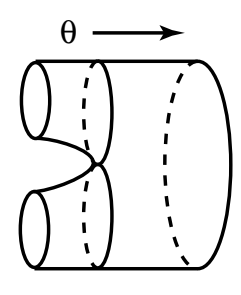

type 1 cc-region

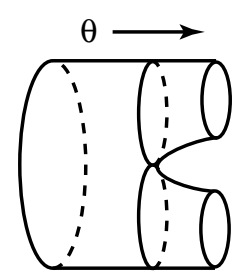

type 2 cc-region

type 1 bc-region type 2 bc-region

Figure 1.13

\section{§. LOCAL CHANGES IN SURFACE DECOMPOSITIONS.}

The surface decompositions which we have just described are not unique. In this section we will describe four ways in which they can be changed. In each of the four cases the possibility of making the change is indicated by examining the combinatorics of the tiling. The change is realized by an isotopy of the surface which is supported in a neighborhood $N$ of a specified small number of basic regions of type $a a, a b, b b, b c$ or $c c$, leaving the surface decomposition unchanged outside $N$.

Our goal, of course, is to simplify the tiling, but that depends upon the particular application, and upon having an appropriate measure of complexity: 
(2.1) The 'change in foliation' move of $\S 2.1$ has been used to reduce the valence of particular vertices in the tiling. See the proofs of Theorem 3.1 and 4.3 for examples of how it has been used to reduce complexity.

(2.2) 'Stabilizing along an $a b$-tile', described in $\S 2.2$, applies to surfaces with boundary. Let $L=\partial \mathcal{F}$ be a closed $n(L)$-braid. Let $V$ be the number of vertices in the tiling of $\mathcal{F}$. Stabilization along an $a b$-tile will be seen to decrease $V$ at the expense of increasing $n(L)$. See Theorem 4.2 for an application.

(2.3) Our 'destabilization' move, described in $§ 2.3$, applies to surfaces with boundary. It will be familiar to most readers: it removes a 'trivial loop' from the closed braid $L$, reducing both $V$ and $n(L)$. See the Corollary to Theorem 4.2 for an application.

(2.4) Our 'exchange move' (which is actually two different but related moves) is discussed in $\S 2.4$. It allows us to detect, by examining the tiling, how to 'empty pockets' and then remove from the surface. It reduces $V$, keeping $n(L)$ fixed. For an application see the proofs of Theorems 3.1 and 4.3 .

\subsection{Changes in foliation.}

The choice of a foliation of $\mathcal{S}$ is not unique, and our first move involves ways in which the surface decomposition can be changed by an isotopy of $\mathcal{S}$ or, equivalently, by an isotopy of the fibers of $H$ keeping $\mathcal{S}$ fixed. We may therefore think of the move as either a change in foliation or a change in fibration. We have chosen to think of it as a change in foliation. This particular change was introduced in [BM 4] for 2-spheres and was modified in BM 5] for certain spanning surfaces. In Theorem 2.1, we extend the move for arbitrary incompressible spanning surfaces and closed surfaces $\mathcal{S}$. As described in $\S 1$, a singularity $s$ in the foliation of $\mathcal{S}$ is positive (respectively negative) if the direction of the positive normal to $\mathcal{S}$ at $s$ agrees (respectively disagrees) with the direction of the normal to $H_{\theta}$ which points in the direction of increasing $\theta$. We label the sign of a singular leaf, surgery, or region, according to the sign of its associated singularity. Two regions in a surface decomposition are adjacent if they have a common boundary arc or boundary circle.

The reader may wish to look ahead to Figure 2.7 to see the singular leaves which correspond to our changes in foliation, described in Theorem 2.1 below, for all possible cases of two regions adjacent at a $b$-arc, when $\mathcal{S}=\mathcal{F}$. 
Theorem 2.1. Suppose that the decomposition of $\mathcal{S}$ contains regions $R_{1}, R_{2}$ of the same sign which are adjacent at a b-arc or c-circle $\gamma$. Then there is an isotopy $\phi$ taking $\mathcal{S}$ to $\mathcal{S}^{\prime}$ such that:

(i) $\phi\left(R_{1} \cup R_{2}\right)=R_{1}^{\prime} \cup R_{2}^{\prime}$, where $R_{1}^{\prime}$ and $R_{2}^{\prime}$ are adjacent regions of the same sign in the decomposition of $\mathcal{S}^{\prime}$.

(ii) The decomposition of $\mathcal{S}^{\prime} \backslash \mathcal{I} \backslash \sqcup\left(\mathcal{R}_{\infty}^{\prime} \cup \mathcal{R}_{\in}^{\prime}\right)$ is the same as the decomposition of $\mathcal{S} \backslash \mathcal{I} \backslash \sqcup\left(\mathcal{R}_{\infty} \cup \mathcal{R}_{\in}\right)$.

(iii) $\phi\left(s_{1}\right)=s_{2}^{\prime}$ and $\phi\left(s_{2}\right)=s_{1}^{\prime}$, where: $s_{i}, s_{i}^{\prime}$ are the singularities contained in regions $R_{i}, R_{i}^{\prime}$, respectively, for $i=1,2$ and $\left(s_{1}, \gamma, s_{2}\right),\left(s_{1}^{\prime}, \gamma^{\prime}, s_{2}^{\prime}\right)$ are their cyclic orders in the fibration relative to the common boundary $\gamma, \gamma^{\prime}$ of their respective regions.

(iv) If $\mathcal{S}=\mathcal{F}$ (so by Theorem 1.2.1 $\gamma$ is a b-arc), $\phi$ decreases the valence of each vertex $v$ and $w$ in $\partial \gamma$ by one.

Proof of Theorem 2.1. In Figures 2.1 (a) and (c) we have illustrated two different embeddings of a subdisk of $R_{1} \cup R_{2}$ in 3-space. The fibers of $H$ are viewed as horizontal planes, and the polar angle function is the height function. Note that each pair of singularities in (a), (c) bounds an arc $\mu$, $\mu^{\prime}$, respectively, in $\mathcal{S}$ which is transverse to the fibration. To visualize the isotopy of $\mathcal{S}$ which takes the embedded disk shown in (a) to the disk shown in (c), pull $\mathcal{S}$ along as the arc $\mu$ is isotoped to the arc $\mu^{\prime}$. Note that the singularities $s_{1}, s_{2}$ which are endpoints of $\mu$ have heights opposite to those of their images $s_{2}^{\prime}, s_{1}^{\prime}$, respectively, under the isotopy. Figure 2.1 (b) shows the intermediate stage of the isotopy at which the endpoints of the image of $\mu$ have the same height. The foliations which correspond to the embeddings of Figure 2.1 are shown directly below them in Figure 2.2. We define a change in foliation as the passage from the embedding in (a) either to the embedding in (c) or to a $120^{\circ}$ rotation of the embedding in (c) about the direction of increasing $\theta$, according to the left hand rule. We will prove that under the given hypotheses there is no obstruction to such a change and that, in the case of regions in the decomposition of a spanning surface adjacent at a $b$-arc $\gamma$, the change decreases the valence of each vertex of $\gamma$ by one.

Note that the embedding in Figure 2.1(c) is obtained from the embedding in Figure 2.1(a) by a $120^{\circ}$ rotation about the direction of increasing $\theta$, according to the left hand rule. Therefore, to show that there is no obstruction to a change in foliation it suffices, by symmetry, to show that there is 
no obstruction to the passage from the embedding in Figure 2.1(a) to the one in Figure 2.1(c).

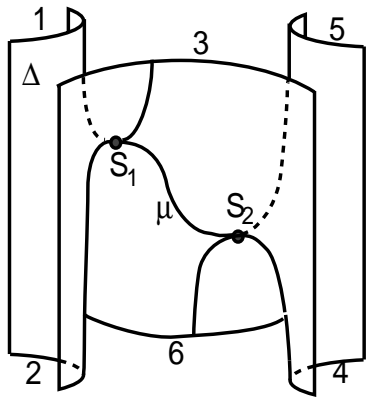

(a)

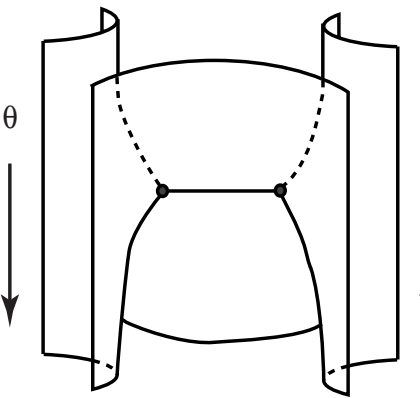

(b)

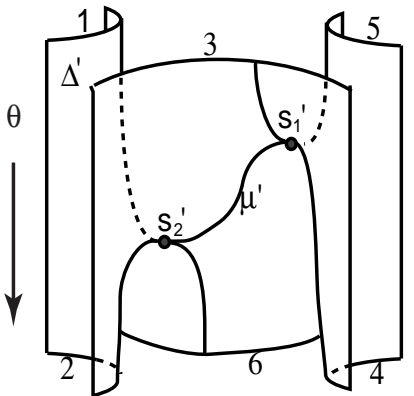

(c)

Figure 2.1

It will be convenient to work in $S^{3}$, so assume that each fiber $H_{\theta}$ is a meridian disk. Up to a choice of orientation of $\mathcal{S}$, the surgeries associated to the singularities $s_{1}, s_{2}$ are shown in Figure 2.3, as we proceed forward through the fibration. Thus, there is a disk $\Delta \subset R_{1} \cup R_{2}$ with embedding as shown in Figure 2.1 (a) and foliation as shown in Figure 2.2 (a). Note that the arc $\mu \subset$ $\Delta$ is contained in the disk $D$ shown in Figure 2.4 (a). If $\operatorname{Int}(D) \cap S=\operatorname{Int}(\mu)$ and $D \cap L=\emptyset$, then we can isotope $\Delta$ to the disk $\Delta^{\prime}$ of Figure 2.1 (c) by pulling $\mathcal{S}$ along in a small neighborhood of $D$ as we push $\mu$ through $D$ to $\mu^{\prime}$ as shown in Figure 2.4. Since this isotopy does not change $\mathcal{S} \backslash \Delta$, it leaves the decomposition of $\mathcal{S} \backslash\left(R_{1} \cup R_{2}\right)$ unchanged as well. Also, since it is the identity on $\mathcal{S} \backslash \Delta$ and since $\Delta^{\prime}$ has the foliation shown in Figure 2.2 (c), it is a simple matter to check that it takes $R_{1} \cup R_{2}$ to $R_{1}^{\prime} \cup R_{2}^{\prime}$, where $R_{1}^{\prime}$ and $R_{2}^{\prime}$ are adjacent regions of the same sign in the decomposition of $\mathcal{S}^{\prime}$. Thus, to prove (i),(ii),(iii) of the theorem, we only need to show that we can first isotope $\mathcal{S}$, without changing its decomposition, so that such a disk $D$ with interior disjoint from $\mathcal{S} \backslash \mu$ and $L$ exists. This isotopy followed by the isotopy from Figure 2.1 (a) to Figure 2.1 (c) is exactly the isotopy $\phi$ described in (i)(ii)(iii) of the theorem.

Note that $\mu$ separates the desired disk $D$ into two subdisks which are identical up to a choice of increasing $\theta$. By symmetry, we need only show that one of the subdisks has interior disjoint from $\mathcal{S}$ and $L$. It therefore suffices to find an arc $\alpha$ as shown in Figures 2.3 and 2.4 which has interior disjoint from $\mathcal{S}$ and $L$, as we proceed through the fibration between the fibers containing $s_{1}$ and $s_{2}$. 


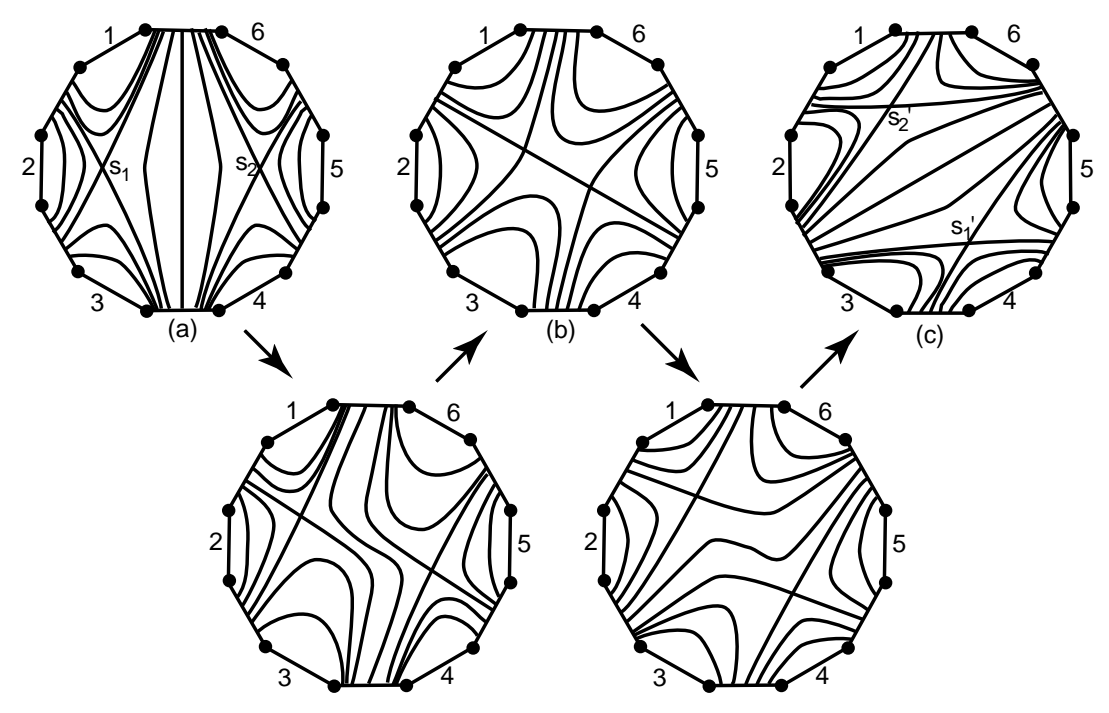

Figure 2.2

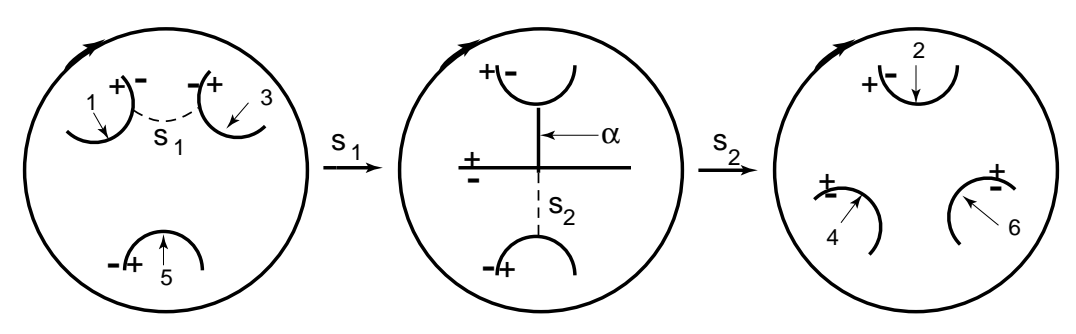

Figure 2.3

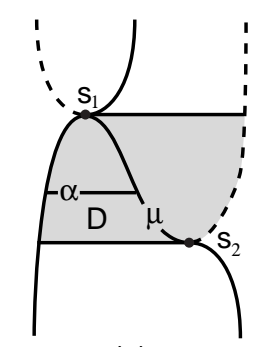

(a)

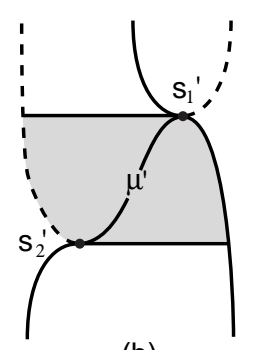

(b)

Figure 2.4 
We first prove the case in which $R_{1}$ and $R_{2}$ are adjacent at a $c$-circle $c$. Up to a choice of orientation of $\mathcal{S}$, we may assume the singularities $s_{1}, s_{2}$ in $R_{1}, R_{2}$ are positive. Let $H_{c}$ be the fiber containing $c$, let $D_{c}$ be the disk in $H_{c}$ bounded by $c$, and let $A_{c}$ be the annulus $H_{c} \backslash D_{c}$. Let $H_{1}$ and $H_{2}$ be the fibers occurring just after $s_{1}$ and $s_{2}$ in the fibration, respectively. We shall not distinguish between $c$ and any circle isotopic to $c$ on $\mathcal{S}$ between $H_{1}$ and $H_{2}$. Since $R_{1}$ and $R_{2}$ are adjacent at a $c$-circle, $R_{1}$ has type $b c$ or type $c c$.

Suppose $R_{1}$ has type $c c$. It has either a type 1 or type 2 embedding in $S^{3} \backslash$ $L$ (see Figure 1.13), and Figure 2.5 (a) and (b) illustrates the corresponding surgeries resulting in the positive singularity $s_{1}$. Suppose that $R_{1}$ has a type 1 embedding. Since $s_{1}$ occurs just before $H_{1}$, there is an arc $\alpha \subset D_{c}$ in $H_{1}$ as shown in Figure 2.5 (a) with interior disjoint from $L$ and $\mathcal{S}$. However the link must (and the surface may) intersect $D_{c} \backslash \alpha$ at this point in the fibration. We must show that we can isotope $\mathcal{S}$, without changing its decomposition, so that the interior of $\alpha$ does not intersect $\mathcal{S}$ or $L$, as we proceed forward through the fibration between $H_{1}$ and $H_{2}$.

(a)

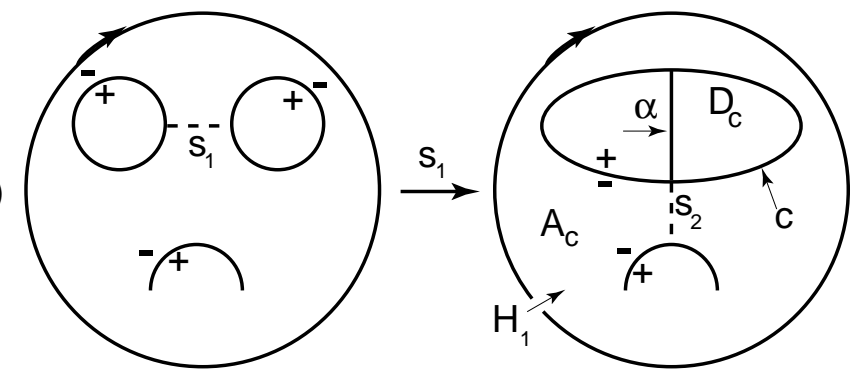

(b)

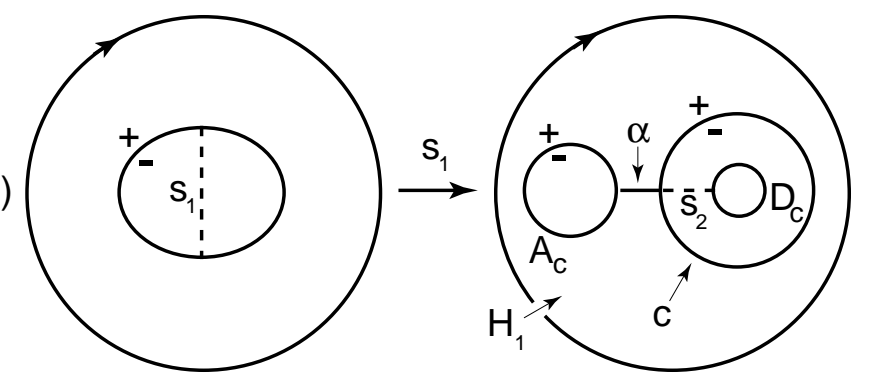

Figure 2.5

Let $X$ be the 3 -ball which is formed by the union of all the disks $D_{c}$ contained in the fibers between $H_{1}$ and $H_{2}$. If no braiding of $L$ or surgeries occur in $X$, then there is nothing to obstruct $\alpha$ as we proceed forward through the fibration between $H_{1}$ and $H_{2}$. Suppose that braiding of $L$ or singularities occur in $X$. Since none of the singularities involve $c$ and since $L \cap c=\emptyset$, we 
can isotope the intersection of the interior of $X$ with $\mathcal{S} \cup \mathcal{L}$ forward in a direction transverse to the fibration, until all braiding and singularities have been pushed out of $X$. Since this isotopy moves $\mathcal{S}$ and $L$ in a direction transverse to each fiber, $L$ remains transverse to the fibration, and the decomposition of that part of $\mathcal{S}$ affected by the isotopy is not disturbed. Afterwards, $\alpha$ can remain fixed as we proceed forward in the fibration through $X$, with its interior disjoint from $L$ and $\mathcal{S}$. Then, since any surgery resulting in a positive $s_{2}$ must be represented by the dotted line in $A_{c}$ shown in Figure 2.5 (a), the singularities and braiding of $L$ which we just pushed forward out of $X$ can be assumed to occur after $s_{2}$ in the fibration. In addition, the singularity $s_{2}$ does not cause $\mathcal{S}$ to intersect the interior of $\alpha$. Thus, we obtain the desired subdisk of $D$ (foliated by $\alpha$ ) with interior disjoint from $\mathcal{S}$ and $L$. Note that this process is only possible when $s_{1}$ and $s_{2}$ have the same sign. If $s_{2}$ has sign opposite to that of $s_{1}$, an attempt to push the braiding of $L$ and singularities of $\mathcal{S}$ forward past $s_{2}$ can result in a link which is not transverse to the fibration and a drastic change in the decomposition of $\mathcal{S}$.

Suppose that $R_{1}$ has a type 2 embedding. There is an $\operatorname{arc} \alpha \subset A_{c}$ in $H_{1}$ as shown in Figure 2.5 (b) with interior disjoint from $L$ and $\mathcal{S}$, and any surgery representing a positive $s_{2}$ is represented by a dotted line in $D_{c}$. Let $X$ be the thickened cylinder formed by the union of all annuli $A_{c}$ between $H_{1}$ and $H_{2}$. As in the type 1 case, none of the singularities in $X$ involve $c$ and $L \cap c=\emptyset$. Therefore we can isotope the intersection of the interior of $X$ with $\mathcal{S} \cup \mathcal{L}$ forward in a direction transverse to the fibration, until all braiding and singularities have been pushed out of $X$, and there is nothing to obstruct $\alpha$ between $H_{1}$ and $H_{2}$. Then, since the surgery corresponding to $s_{2}$ must be represented by a dotted line in $D_{c}$, we can push the braiding of $L$ and singularities of $\mathcal{S}$ forward in the fibration past $s_{2}$. As in the preceding case, the singularity $s_{2}$ does not cause $\mathcal{S}$ to intersect $\alpha$, and we again obtain the desired subdisk of $D$ with interior disjoint from $\mathcal{S}$ and $L$.

If $R_{1}$ has type $b c$, then it must have a type 1 embedding, since otherwise it is not adjacent to $R_{2}$ at a $c$-circle. The desired subdisk of $D$ is obtained in this case by essentially the same proof as for the case in which $R_{1}$ has type $c c$ and a type 2 embedding. This completes the proof of the theorem in the case in which $R_{1}$ and $R_{2}$ are adjacent at a $c$-circle.

The proof of (i)(ii)(iii) in the case in which $R_{1}$ and $R_{2}$ are adjacent at a $b$-arc is similar in flavor to the previous case. Suppose that $R_{1}$ and $R_{2}$ are adjacent at a $b$-arc $\beta$ with vertices $v$ and $w$. In this case, we have the situation shown in Figure 2.6, as we proceed forward in the fibration. As in the previous case, let $H_{1}$ and $H_{2}$ be the fibers which occur just after the singularities $s_{1}$ and $s_{2}$, respectively. Again, there is an arc $\alpha$ in $H_{1}$ which does not intersect $\mathcal{S}$ or $L$ (see Figure 2.6). We must show that $\alpha$ 
does not intersect $\mathcal{S}$ or $L$, as we proceed forward through the fibration from $H_{1}$ to $H_{2}$. Note that the arc $\beta$ separates $H_{1}$ into two disks. Let $D_{\alpha}$ be the disk which contains $\alpha$ (it is shaded in Figure 2.6). Since $\beta$ does not surger between $H_{1}$ and $H_{2}$, there is such a disk in each fiber between $H_{1}$ and $H_{2}$. Let $X$ be the 3-ball which is formed by the union of all the disks $D_{\alpha}$. If no singularities or braiding of $L$ occur in $X$, then $\alpha$ can remain fixed in $D_{\alpha}$ with interior disjoint from $\mathcal{S}$ and $L$ as we proceed forward in the fibration through $X$. Since $\beta \cap L=\emptyset$ and since none of the singularities which may occur between $H_{1}$ and $H_{2}$ involve $\beta$, we can push all singularities and braiding of $L$ forward in the fibration out of the 3-ball $X$ as in the case when the regions are adjacent at a $c$-circle. Now note that the surgery corresponding to $s_{2}$ is represented by a dotted arc in $H_{1} \backslash D_{\alpha}$. Thus, we can push the braiding of $L$ and singularities of $\mathcal{S}$ forward in the fibration past $H_{2}$, and $s_{2}$ does not cause $\mathcal{S}$ to intersect $\alpha$ or force $L$ to a position that is not transverse to the fibration. As in the $c$-circle case, the desired subdisk of $D$ with interior disjoint from $\mathcal{S}$ and $L$ is foliated by the $\operatorname{arcs} \alpha$. This completes the proof of (i)(ii)(iii) of the theorem.

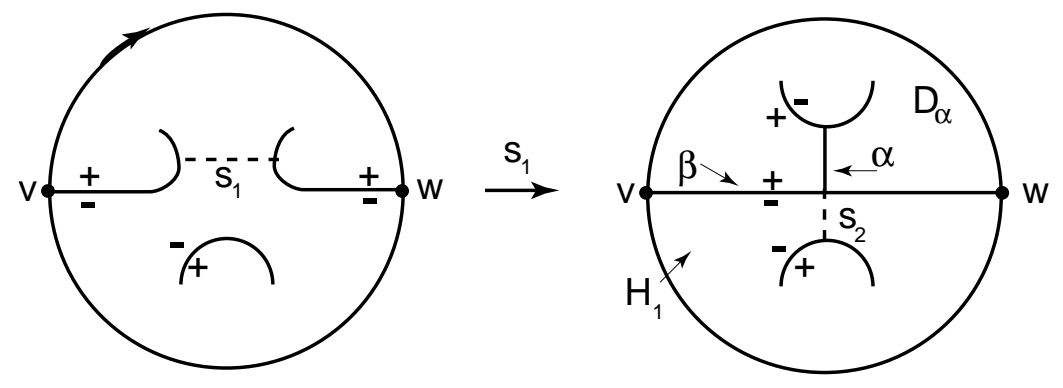

Figure 2.6

Our proof of (iv) requires a careful inspection of the region foliations before and after the isotopy $\phi$. Note that, since $\mathcal{S}=\mathcal{F}$ is a spanning surface, any two regions adjacent at a $b$-arc $\gamma$ must have type $b b$ or $a b$. This yields three cases, up to symmetry, for the types of $R_{1}$ and $R_{2}$ : (1) both are $b b$-tiles; (2) $R_{1}$ is a $b b$-tile and $R_{2}$ is an $a b$-tile or (3) both are $a b$-tiles. We only need to show that the singular leaves corresponding to the change in foliations for these three cases are as shown in (1), (2) and (3) of Figure 2.7, respectively, since each of these figures illustrates the required reduction in valence for the vertices $v$ and $w$ of $\gamma$. The first column after the arrows in Figure 2.7 illustrates the result of a change in foliation as described in Figure 2.1. The second column after the arrows shows the result of the second type of change in foliation, i.e., the passage from Figure 2.1(a) to a $120^{\circ}$ rotation of Figure 
2.1(c). Since the proofs are identical, we will only show that the singular leaves in the first column after the arrows are the result of the change in foliation shown in Figure 2.1.

(1)

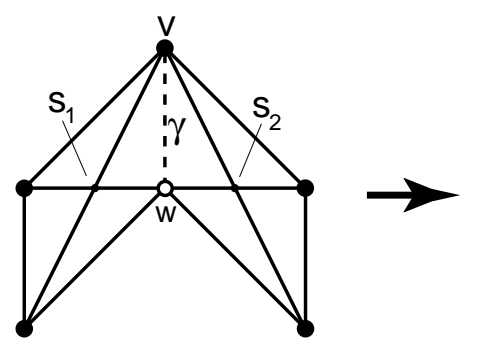

(2)

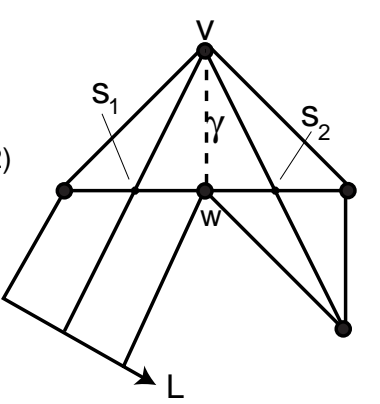

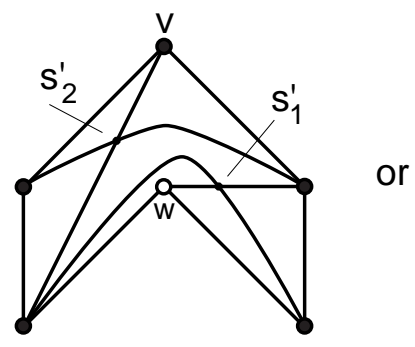
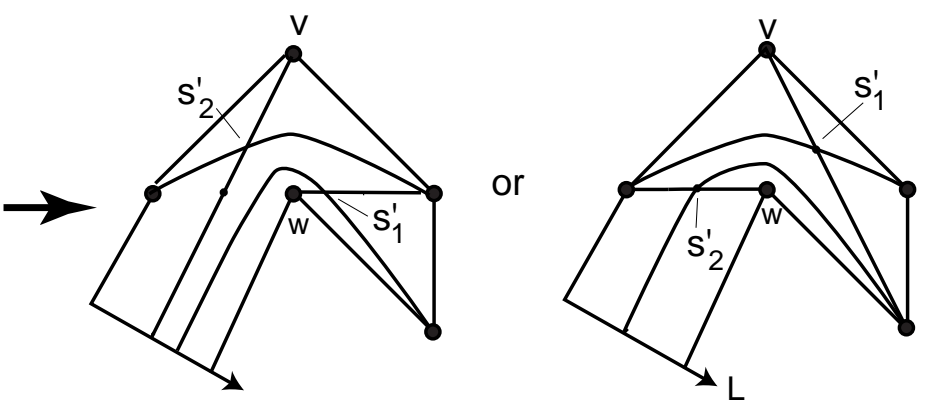

(3)
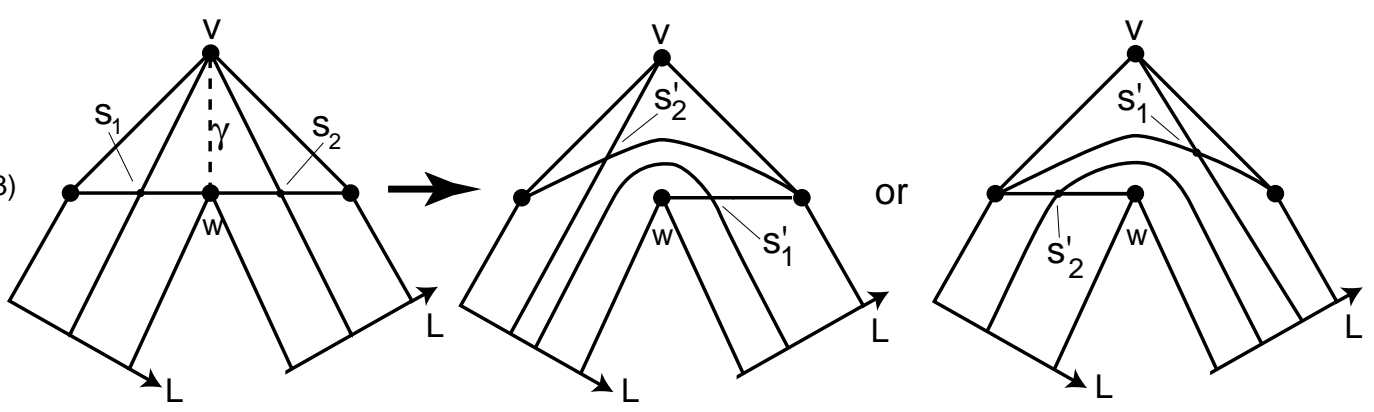

Figure 2.7

Suppose we are in case (1). Thus $R_{1}$ and $R_{2}$ are two $b b$-tiles adjacent at a $b$-arc with vertices $v$ and $w$. Note that the arcs labeled $1,2,3,4,5,6$ in the boundary of $\Delta$ in Figure 2.2 (a) are portions of $b$-arcs, and the unlabeled arcs are transverse to the foliation. Therefore, in order for the leaves in the foliation to match up, $\Delta$ must be situated in $R_{1} \cup R_{2}$ as shown in Figure 2.8 (a). Since $\phi$ does not change the foliation of $R_{1} \cup R_{2}$ outside $\Delta$, the foliation of $R_{1}^{\prime} \cup R_{2}^{\prime}$ can be obtained by replacing the foliation of $\Delta$ with that of $\Delta^{\prime}$. 
Thus, $R_{1}^{\prime} \cup R_{2}^{\prime}$ is foliated as shown in Figure 2.8 (b). The singular leaves corresponding to a change in foliation in case (1) are therefore as shown in Figure 2.7 (1). This concludes the proof of (iv) in case (1). The proof for cases (2) and (3) is identical. Using Remark 1.1, the reader will be able to see immediately that the foliation changes resulting from $\phi$ in these cases are as shown in Figures 2.7 (2) and (3), respectively. This concludes the proof of the theorem. \|

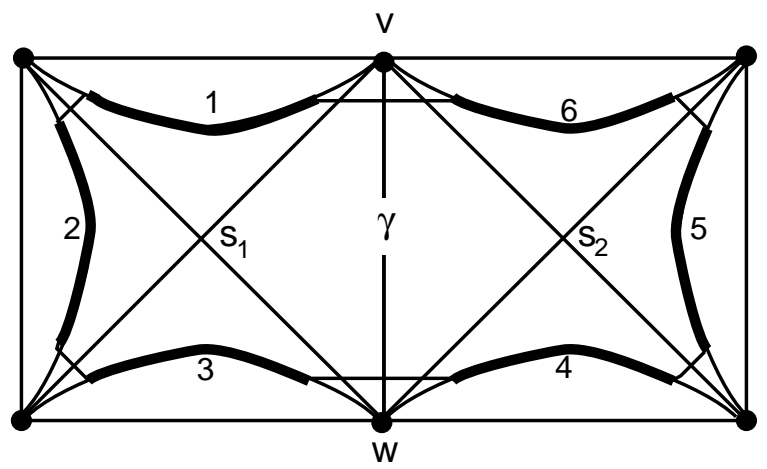

(a)

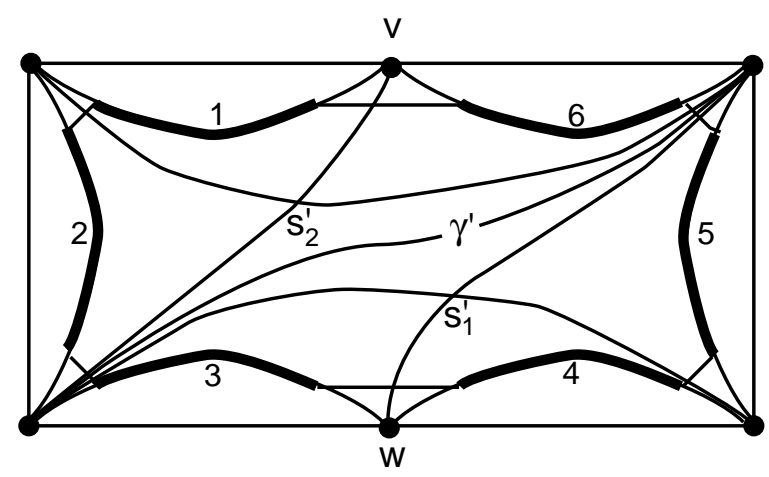

(b)

Figure 2.8

Remark 2.1 The reader can use the same method which is used in the proof of Theorem 2.1 (iv) to check that if a vertex $w$ has type $(a, b)$ and sign $( \pm, \pm)$ then, after a change in foliation, $w$ has type $(a)$. The singular leaves corresponding to this change in foliation are shown in Figure 2.9. 


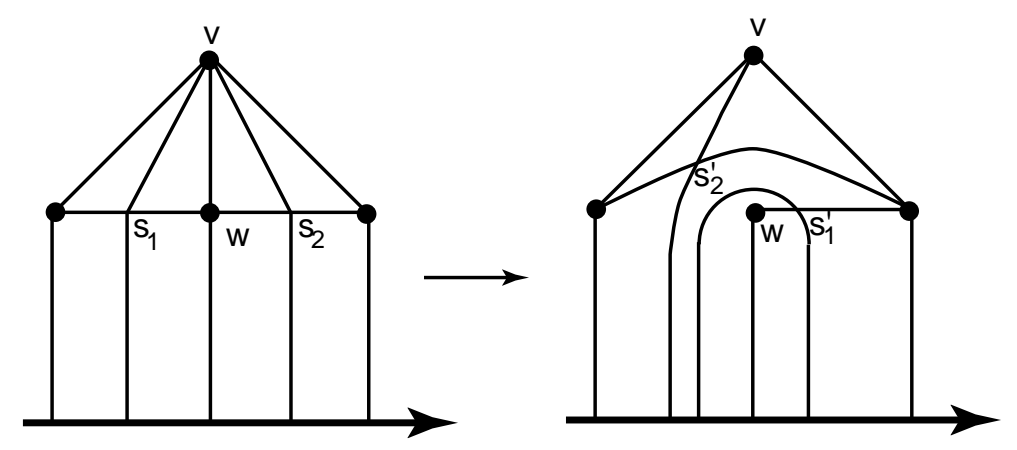

Figure 2.9

\subsection{Stabilization along $a b$-tiles.}

In this subsection and the next we will describe two modifications for an incompressible spanning surface $\mathcal{F}$ which change the braid representative $L$ and the tiling, but do not change the link type $\mathcal{L}$. Both of these moves change the braid index. They were, in effect, introduced by Bennequin in [Be]. The "stabilization move" which we describe in this subsection adds a trivial loop to the braid representative $L$; and the "destabilization move" which we consider in the next subsection deletes one. In this respect, the two moves are mutually inverse with respect to the closed braid structure on $\mathcal{L}$. However, they are not mutually inverse with respect to their effect on the tiling of $\mathcal{F}$. Indeed, the stabilization move results in the elimination of a negative vertex whereas the inverse of the destabilization move results in the addition of a positive vertex. The stabilization move deletes an $a b$-tile, and changes nearby tile types. The inverse of the destabilization move, adds an $a a$-tile, and leaves the rest of the tiling unchanged.

The modification in the tiling of $\mathcal{F}$ which we call stabilization along an $a b$-tile, is shown in Figure 2.10(a). It is realized by pushing a subarc $\alpha$ of $L$ along a disc neighborhood of part of the singular leaf in an $a b$-tile $T$. The boundary of the neighborhood may be chosen to be transverse to leaves of the foliation and to the fibration, so the result is a new closed braid representative $L^{\prime}$ of the link, bounding a new surface $\mathcal{F}^{\prime}$ which is tiled, but with one less tile than $\mathcal{F}$ and one less negative vertex. In fact, letting $N$ be the union of all tiles of $\mathcal{F}$ with $v$ as a vertex, we can summarize the effect of stabilizing along the $a b$-tile $T$ as follows:

(1) $T$ and its negative vertex are deleted. 
(2) Any $a b$-tile in $N \backslash T$ is transformed into an $a a$-tile.

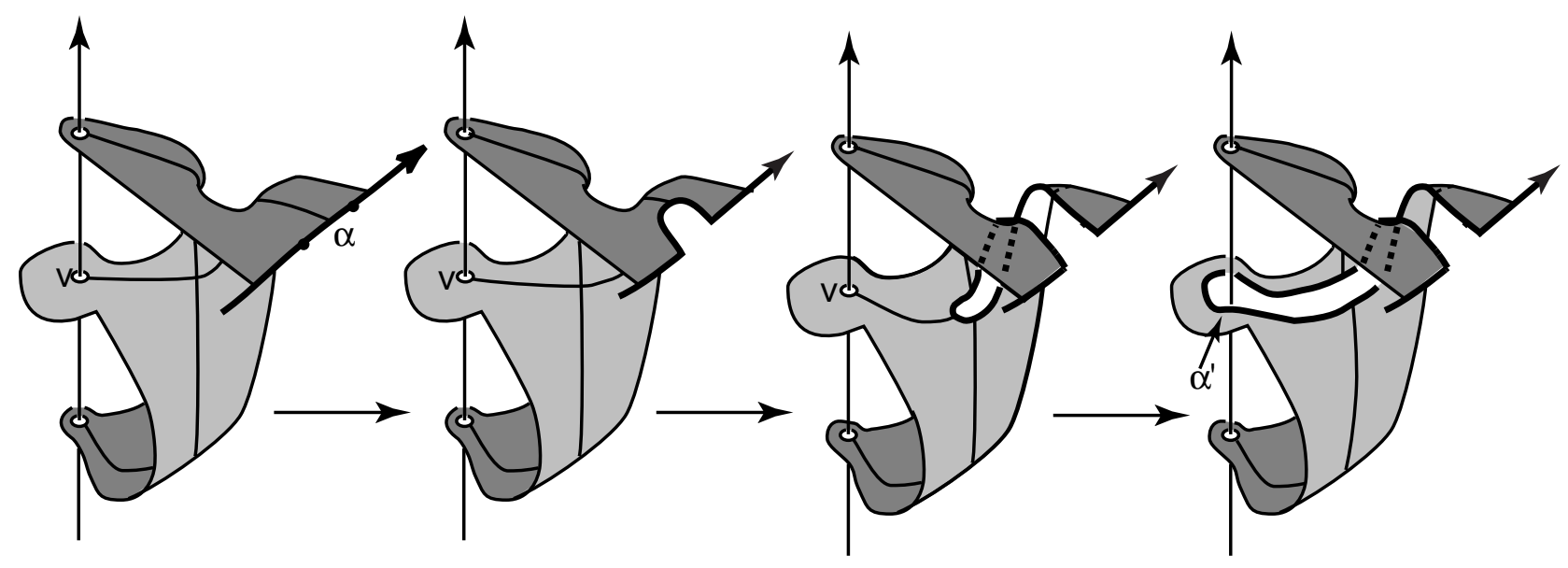

(a) Stabilization along an ab tile, viewed on the embedded tile

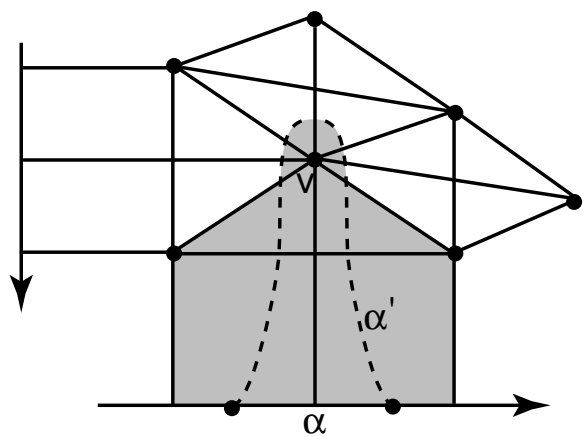

(b) viewed in the foliation

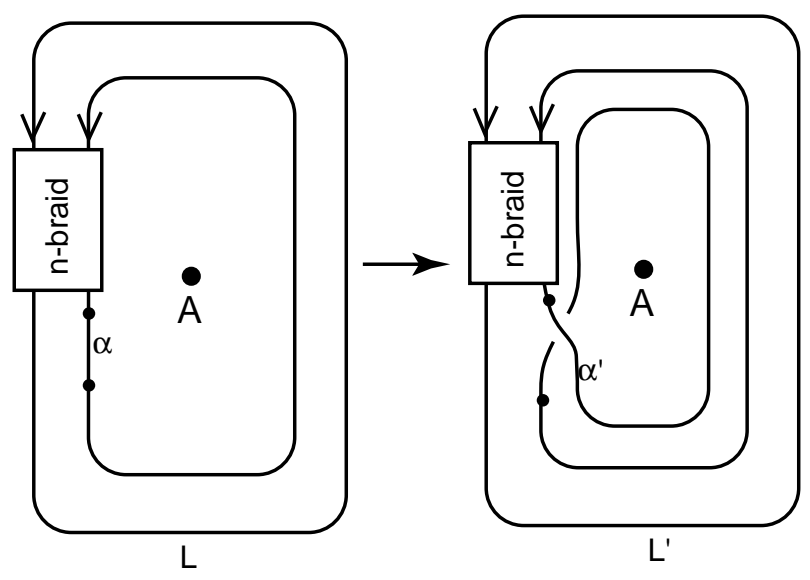

(c) Viewed in the projection

Figure 2.10

(3) Any $b b$-tile in $N$ is transformed into an $a b$-tile.

(4) The tiling of $\mathcal{F}^{\prime}$ is the same as the tiling of $\mathcal{F}$ outside $N$.

(5) The resulting braid representative $L^{\prime}$ is obtained from $L$ by the addition of a trivial loop, as shown in Figure 2.10(c). This increases the braid index by one. 
To see $(1)(2)(3)(4)$, examine Figure 2.10(b), which shows one sample $N$, flattened out. The change in braid representative described in (5) can be seen by noting that Figure 2.10(c) is just a projection parallel to the axis of the link described in Figure 2.10(a).

\subsection{Destabilization via a type $(a)$ vertex.}

We will now show that whenever there is a vertex of valence one in the tiling of $\mathcal{F}$, then it has type $(a)$, and we can modify the tiling in the manner shown in Figures 2.11 (a) and (b). We call this modification destabilization via a type (a) vertex.

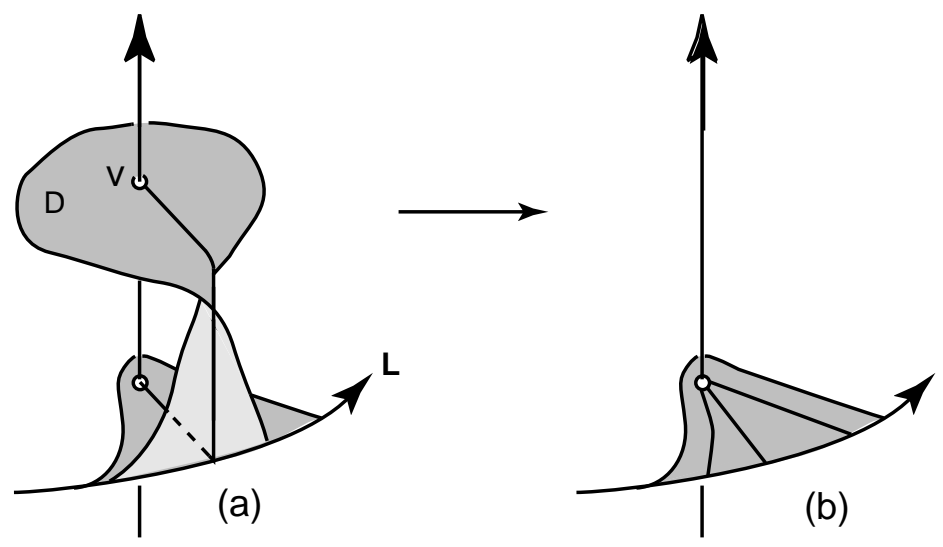

Destabilization via a type a vertex

Figure 2.11

A vertex $v$ of valence 1 in the tiling occurs when two of the edges in a single tile $T$ are identified in $\mathcal{F}$. Such a vertex must have type $(a)$. For if it has type $(b)$, the only other possibility, then there is a type $b b$ (respectively $a b$ ) tile in the tiling of $\mathcal{F}$ which has two adjacent $b$-edges identified. This requires that $T$ have only 3 (respectively 2 ) distinct vertices instead of 4 (respectively 3 ). The tile $T$ contains exactly one singular point, and this singularity occurs when two non-singular leaves come together. This means that just before the singularity there were two components of $H_{\theta} \cap \mathcal{F}$ emerging from a single point of $A \cap \mathcal{F}$, which is impossible. Thus $v$ has type (a) and, since the only tile which has a pair of adjacent $a$-edges meeting at a common vertex is an $a a$-tile, it follows that $T$ is $a a$-tile.

We know there is exactly one canonical embedding for an $a a$-tile in 3space, shown in Figure 1.13. Identifying two edges of an $a a$-tile with end- 
points on a common vertex therefore yields the canonical embedding in 3space for $T$ shown in Figure 2.11 (a). Notice that there is a radially foliated disk $D$ in $T$ cut off from $\mathcal{F}$ by the arc of the singular leaf of $T$ with both endpoints on $L$. Therefore, there must be a trivial loop in the braid representation of $L$. Destabilization via the type $(a)$ vertex $v$ is realized by pushing $L$ through $D$ to the singular leaf in $T$, and then modifying this new link representative so that it becomes transverse to all fibers of $H$ (see Figure 2.11 (b)). The result is a new closed braid representative $L^{\prime}$ bounding a new surface $\mathcal{F}^{\prime}$, with changes summarized as follows:

(1) The $a a$-tile $T$ and its type $(a)$ vertex $v$ are deleted.

(2) The tiling of $\mathcal{F}^{\prime}$ is the same as the tiling of $\mathcal{F}$ outside $T$.

(3) $L^{\prime}$ is obtained from $L$ by deleting a trivial loop, which decreases the braid index by one.

\subsection{Exchange Moves.}

The modification which was described in $\S 2.3$ was based upon the existence of a vertex of valence 1 in the tiling. In this subsection we consider modifications which are based upon the existence of a vertex of valence 2 .

The precise definition of our exchange move (given in Theorem 2.2 below) is based upon the tiling of our surface, however the reader may find it easier to understand the move by looking first at its effect on a knot diagram. See Figure 2.12 for an example. The labels $n_{j}$ on the strands are weights, indicating $n_{j}$ parallel strands. We allow any type of braiding on the $n_{1}+n_{2}$ (respectively $n_{1}+n_{3}$ ) strands in the boxes which are labeled $X$ (respectively $Y)$. Assume that the braid axis $A$ is the $z$-axis, and that the arc which is labeled $n_{3}$ lies in the $x y$-plane. Up to isotopy of $S^{3}$, an exchange involves an isotopy of $L$ which moves the arc which is labeled $n_{2}$ from a position which is a little bit above (or below) the $x y$-plane to a position which is a little bit below (or above) the $x y$-plane. However, as we shall see, this is only part of the story, because all of our moves are guided by the foliation of the surface, and exchange moves are too.

We shall define exchange moves in two different situations (parts (2.2.1) and (2.2.2) of Theorem 2.2). The phenomena will seem, initially, to be very different in the two cases, but after the proofs are complete we will be able to understand why they are in fact very closely related. 


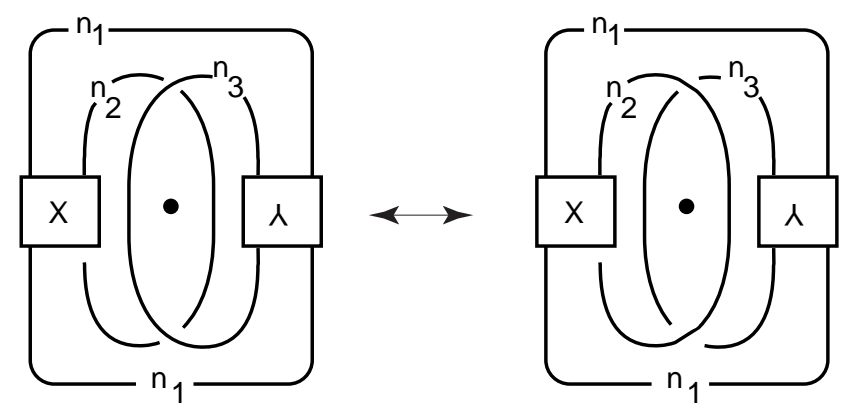

\section{Changes in the braid during an exchange move}

Figure 2.12

\section{Theorem 2.2 BM 4, BM 5]}

(2.2.1) Suppose that $\mathcal{F}$ has a vertex $v$ of valence 2 , type $(a, b)$ and sign $( \pm, \mp)$. Then $L=\partial \mathcal{F}$ admits an isotopy to a new closed braid $L^{\prime}$, such that:

(a) The isotopy from $L$ to $L^{\prime}$ is a push across a disc $\Delta \subset \mathcal{F}$, where $\Delta$ is a neighborhood of a disc $\delta$ which is contained in the union of two ab tiles in the decomposition of $\mathcal{F}$.

(b) The decompositions of $\mathcal{F}$ and $\mathcal{F}^{\prime}=\mathcal{F} \backslash \cdot$ are identical everywhere except on $\Delta$. The tiling of $\mathcal{F}^{\prime}$ has two fewer vertices than the tiling of $\mathcal{F}$. The braid indices of $L$ and $L^{\prime}$ coincide.

(c) The isotopy $L \rightarrow L^{\prime}$ modifies $L$ near $A$ as depicted in Figure 2.12.

(2.2.2) Suppose that $\mathcal{S}$ has a vertex $v$ of valence 2 , type $(b, b)$ and sign $( \pm, \mp)$. Then $L$ admits an isotopy to a new closed braid $L^{\prime}$ and $\mathcal{S}$ admits an isotopy to a new surface $\mathcal{S}^{\prime}$ such that:

(a) The decompositions of $\mathcal{S}$ and $\mathcal{S}^{\prime}$ are identical except on the two given bb tiles in $\mathcal{S}$. The tiling of $\mathcal{S}^{\prime}$ has two fewer vertices and two fewer singular points than the tiling of $\mathcal{S}$. The braid indices of $L$ and $L^{\prime}$ coincide.

(b) The isotopy $L \rightarrow L^{\prime}$ modifies $L$ near $A$ as as depicted in Figure 2.12.

Proof of Theorem 2.2, part (2.2.1): (See page 597 of [BM 5]) A vertex of type $(a, b)$ occurs when two $a b$-tiles $T_{1}$ and $T_{2}$ are identified along corresponding $a$ and $b$ edges, as in Figure 2.13(a). The vertex $v$ is joined to $\partial \mathcal{F}$ 
by the common $a$-edge in $T_{1}$ and $T_{2}$ and to a second common vertex $w$ by the common $b$-edge.

Let $\delta$ be the disc in $T_{1} \cup T_{2}$ which is cut off by the portions of the singular leaves which connect $w$ to $L$ in $T_{1} \cup T_{2}$. We know the foliations of our two tiles from Figure 1.8, and we know that a neighborhood of $w$ is radially foliated. We may then find an arc $\alpha^{\prime}$ (see Figure 2.13(a)) which begins and ends on $L$ and is everywhere transverse to leaves of the foliation, which cobounds with a subarc $\alpha$ of $L$ a neighborhood $\Delta$ of $\delta$ on $\mathcal{F}$. The disc $\Delta$ is shaded in lightly in Figure 2.13(a). Push $\alpha$ through $\Delta$ to $\alpha^{\prime}$, crossing the axis twice in the process, to obtain a new closed braid representative $L^{\prime}$ of $\mathcal{L}$ in $S^{3}$. The new representative $L^{\prime}$ bounds a new surface $\mathcal{F}^{\prime}$, isotopic to $\mathcal{F}$. The new surface $\mathcal{F}^{\prime}$ is also tiled, but has two fewer tiles and two fewer vertices than the tiling of $\mathcal{F}$. In fact, if $\mathcal{R}$ is the union of our two $a b$-tiles together with all tiles in the decomposition of $\mathcal{F}$ having $w$ as a vertex, then the decomposition of $\mathcal{F}^{\prime}$ is the same as the decomposition of $\mathcal{F}$ outside $\mathcal{R}$. An examination of Figure 2.13(a) shows that the changes in the decomposition on $\mathcal{R}$ are as follows: our two $a b$ tiles are deleted; (2) any other $a b$-tile in $\mathcal{R}$ becomes an $a a$-tile and (3) any $b b$-tile in $\mathcal{R}$ becomes an $a b$-tile.

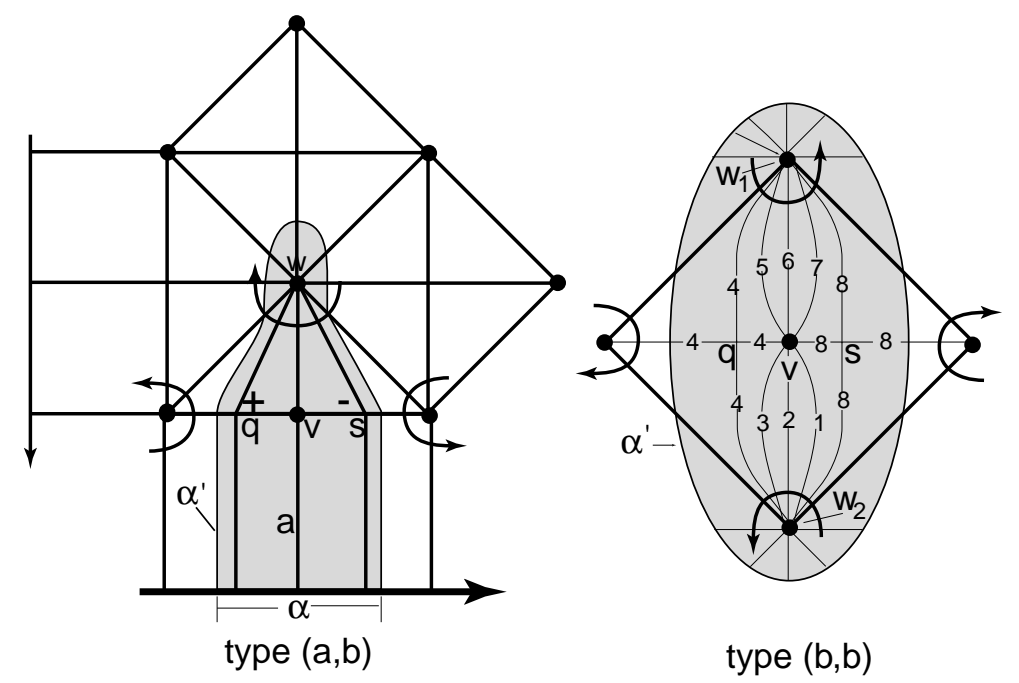

Figure 2.13

Note that the sense of rotation of the flow is in opposite directions about the vertices $v$ and $w$. Thus, the algebraic rotation number of the flow about the deleted vertices is zero, so the algebraic rotation number of $L^{\prime}$ about $A$ is the same as that of $L$, namely $n$. From this it follows that the braid index of $L^{\prime}$ is $n$, because $L^{\prime}$ is transverse to every fiber of $H$.

It only remains to show that $L$ and $L^{\prime}$ are related by an exchange move. 
With this in mind, we study the embedding of $\Delta$ in $\mathbb{R}^{3}$. Since $v$ has sign $( \pm, \mp)$, the two singularities in $\Delta$ have opposite signs. Thus, since the flow is in opposite directions about the vertices $v$ and $w$, it follows that $\Delta$ must be embedded as shown in Figure 2.14(a). Now since all $b$-arcs in the foliation are essential, the link itself must be an obstruction to their removal, as we have shown in Figure 2.14(a). We can push all braiding away from $A$, into the box labeled $X$. Projection parallel to $A$ now clearly shows that when we push $\alpha$ through $\Delta$ to $\alpha^{\prime}$, we are doing an exchange move. In this case $n_{3}=1$.
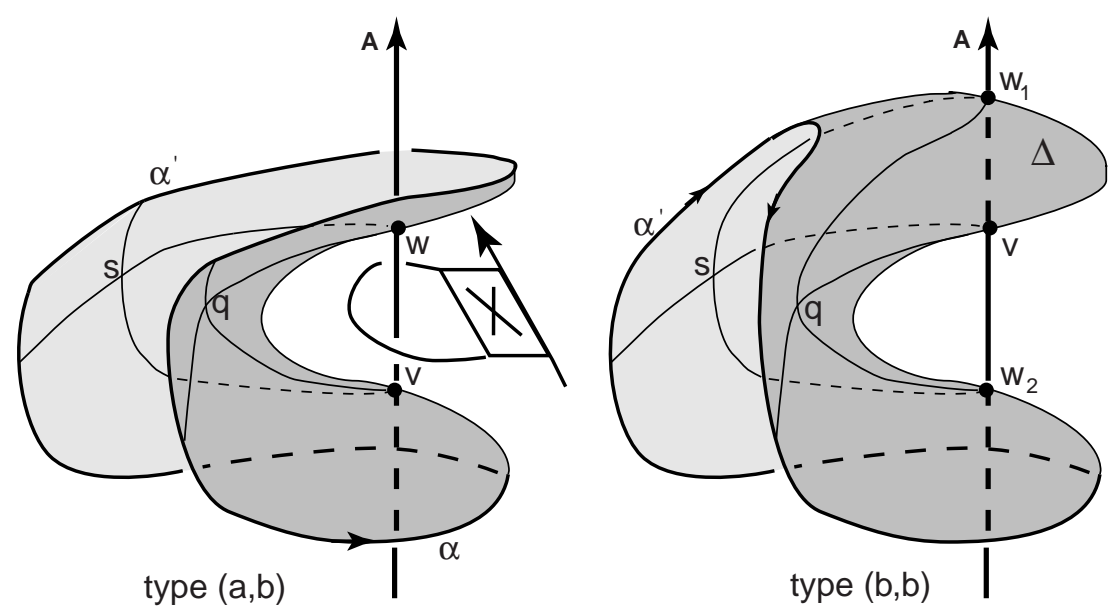

Figure 2.14

Proof of (2.2.2): (See page 130 of [BM 4] $)$. We now suppose that $v$ has sign $( \pm, \mp)$ and type $(b, b)$. The geometry in $(2.2 .2)$ is in many ways similar to that in (2.2.1), because an $a b$ tile may be thought of as having been obtained from a $b b$ tile by deleting a neighborhood of a vertex. However, in (2.2.2) the region near $v$ is in the interior of $\mathcal{F}$, whereas we will be modifying $L$. The modifications are suggested by Figure 2.15.

In order to justify this picture and prove $(2.2 .2)$ we will need to:

1. Show that $\mathcal{S}$ and $L$ are positioned as in the left picture in Figure 2.15;

2. Find structures in the foliation of the two $b b$ tiles which meet at $v$ which will tell us how to modify $L$ to the middle picture in Figure 2.15. We already know that $a b$ tiles are like $b b$ tiles with a deleted vertex, and the structures will appear out of a "shadow" of our two $b b$ tiles; 
3. Show that the decomposition of $\mathcal{S}$ is left invariant under the modification, even when $\mathcal{S}$ is a spanning surface and $L$ its boundary;

4. Show that, on the other hand, the order in which tiles on the surface are intersected by $A$ is changed;

5. Show that the change in the braid structure of $L$ is an exchange move, as illustrated in Figure 2.12, and finally;

6. Show that after the change we have inessential $b$ arcs which can be removed by an isotopy of $\mathcal{S}$ to $\mathcal{S}^{\prime}$ in the right picture in Figure 2.15.

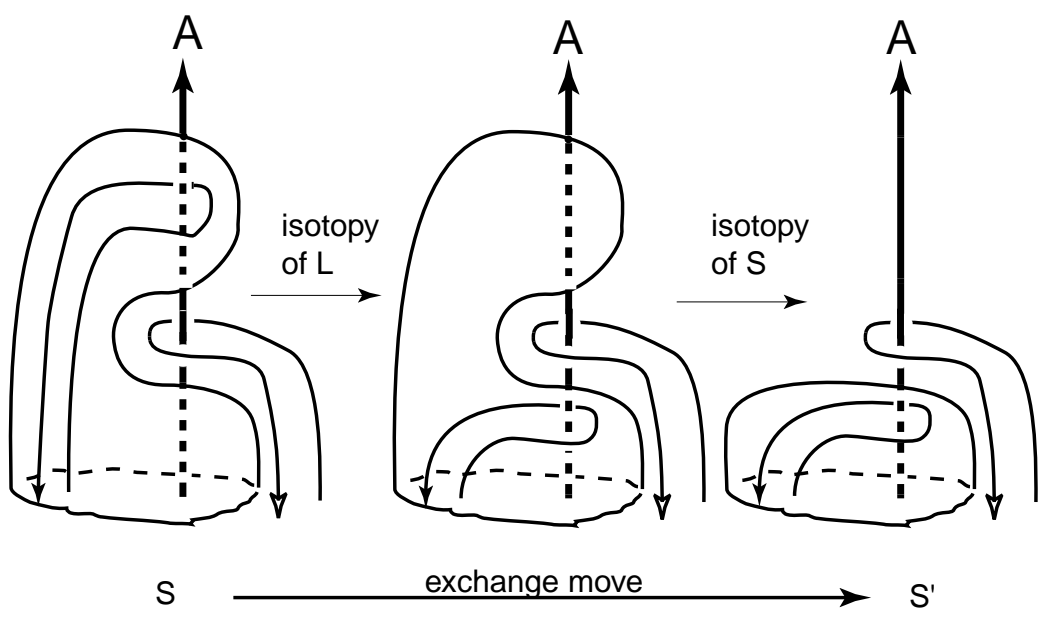

Figure 2.15

Let $T_{1}$ and $T_{2}$ be our two $b b$ tiles and let $q$ and $s$ be the singular points of the two regions which meet at $v$. Let $w_{1}$ and $w_{2}$ be the other vertices of the two edges which meet at $v$. See Figure 2.13(b), which will seem easy because of the analogy with Figure 2.13(a). We have only made one small change in the passage from Figure 2.13(a) to 2.13(b), i.e. we have changed the signs of the vertices to stress the fact that the given vertex of type $(b, b)$ and sign $( \pm, \mp)$ could be either positive or negative, whereas in the case of a vertex of type $(a, b)$, i.e. as in Figure 2.13(a), it is always positive. Portions of the singular leaves which connect $w_{1}$ to $w_{2}$ fit together to cut off a disc $\delta$ on $\mathcal{S}$, and we have sketched in a neighborhood $\Delta$ of $\delta$ on $\mathcal{S}$, chosen so that $\partial \Delta$ is everywhere transverse to the foliation. Notice that the gradient flow on $\mathcal{S}$ is always oriented in opposite senses around the two endpoints of each leaf which joins two points of $A \cap \mathcal{S}$. This implies that $\partial \Delta$ 
has algebraic rotation number 1 about the axis $A$. Since $\partial \Delta$ is everywhere transverse to the foliation, it then follows that $\partial \Delta$ is a 1-braid. The axis $A$ pierces $\Delta$ from alternating sides at $v, w_{1}$ and $w_{2}$. Using this and the fact that $s$ and $q$ have opposite signs, the reader should have no trouble using the procedure described in $\S 1$ to verify that $\Delta$ must be embedded as shown in Figure 2.14(b).

We will need to study this embedding in some detail, and so it will be convenient to assign numbers $0 \leq \theta_{1}<\theta_{2}<\ldots<\theta_{8}<2 \pi$ to the leaves in the foliation of $\Delta$, as in Figure 2.13(b), where the label " $i$ " means $\theta_{i}$. We need to use these leaves to create a region in 3 -space in which to describe the exchange move and the isotopy of $\mathcal{S}$ in a controlled fashion.

Each non-singular $H_{\theta}$ meets $\mathcal{S}$ in a unique arc $b(\theta)$ which joins $v$ to $w_{1}$ or $w_{2}$ and cobounds with a part of the axis $A$ two discs in the fiber $H_{\theta}$. If $b(\theta)$ joins $v$ to $w_{1}$ (resp. $w_{2}$ ), let $\mu_{\theta}$ be the disc which does not contain $w_{2}$ (resp. $w_{1}$ ). We have sketched in one such arc $b(\theta)$ and shaded in the disc $\mu_{\theta}$ in Figure 2.16. If we think of the subsurface $\Delta$ of Figure 2.16 as a slit-open boxing glove, the discs $\mu_{\theta}$ for $\theta_{1}<\theta<\theta_{5}$ will appear to be "outside" the glove, whereas those for $\theta_{5}<\theta<\theta_{1}$ will be "inside" the glove. The disc $\delta$, which is on the glove, is a limiting position for both families of discs. Thus the closure of the union of all of the discs $\mu_{\theta}, \theta \in[0,2 \pi]$ will be two 3-balls $B_{1}$ and $B_{2}$, which intersect along a single arc in the disc $\delta$ of Figure 2.13(b), i.e. the arc which runs from $q$ to $s$ through $v$. The arc $b\left(\theta_{7}\right)$ in Figure 2.17 is in the boundary of $B_{1} \cup B_{2}$.

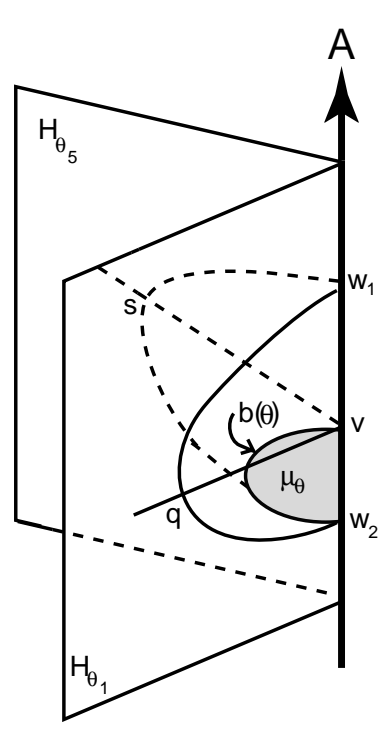

Figure 2.16

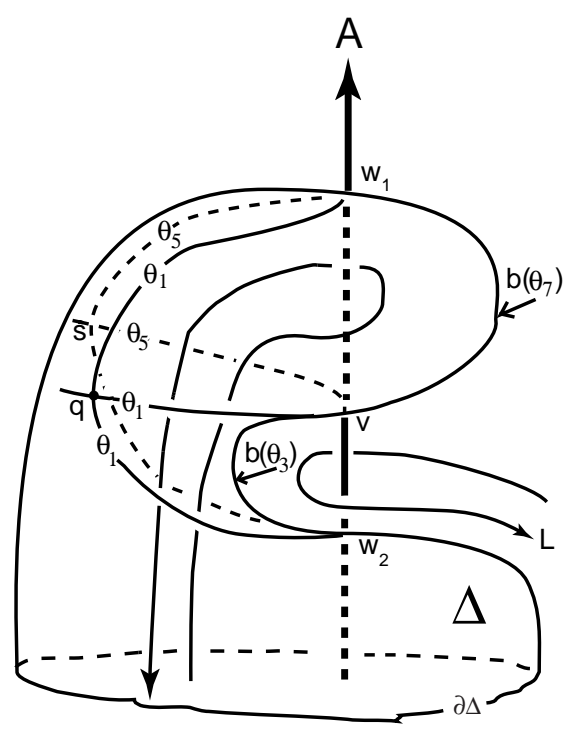

Figure 2.17 
We may assume that $B_{1} \cup B_{2}$ intersects the link $L$ in two unbraided weighted arcs. This is clearly possible, since all braiding may be pushed out of $B_{1} \cup B_{2}$. Notice that the surface $\mathcal{S}$ may meet $B_{1}$ and $B_{2}$ in some number of sheets which are locally parallel to the embedded disc $\Delta$ which we depicted in Figure 2.16. In particular, $\mathcal{S}$ may meet a neighborhood $N_{A}$ of $A$ in some number of radially foliated discs between the discs at $w_{1}$ and $w_{2}$.

Now we need to thicken $B_{1} \cup B_{2}$ a little bit. With this in mind, reparametrize the interval $[0,2 \pi]$ so that the singularities occur at $\theta_{1}=0$ and $\theta_{5}=\pi$. We can then pair the discs $\mu_{\theta}$ and $\mu_{\theta+\pi}$, so that $\mu_{\theta}$ is in $B_{1}$ and $\mu_{\theta+\pi}$ is in $B_{2}$. See Figure 2.18, which depicts subsets of $H_{\theta} \cup H_{\theta+\pi}$. Now, letting $0<\theta<\pi$, notice (see Figure 2.13(b)) that if $H_{\theta}$ is non-singular, then $H_{\theta}$ contains two leaves in the foliation of $\Delta$ : the leaf $b(\theta)$, which joins $v$ to $w_{2}$, and also a small arc $\alpha(\theta)$ which runs out from $w_{1}$ to the boundary of $\Delta$. Similarly, any non-singular fiber $H_{\theta+\pi}$ contains the leaf $b(\theta+\pi)$, which joins $v$ to $w_{1}$, and a small arc $\alpha(\theta+\pi)$ which runs out to the boundary of $\Delta$ from $w_{2}$. Let $N(\theta)$ be a neighborhood of $\mu_{\theta} \cup \mu_{\theta+\pi} \cup \alpha(\theta) \cup \alpha(\theta+\pi)$ in $H_{\theta} \cup H_{\theta+\pi}$, chosen so that $N(\theta) \cap L=\left(\mu_{\theta} \cup \mu_{\theta+\pi}\right) \cap L$ and $N(\theta) \cap \mathcal{S}$ is the union of $\left(\mu_{\theta} \cup \mu_{\theta+\pi}\right) \cap \mathcal{S}$ and $\left(H_{\theta} \cup H_{\theta+\pi}\right) \cap N_{A}$. Finally, choose the neighborhoods $N(\theta)$ so that they vary smoothly as $\theta$ is varied between 0 and $\pi$. Let $N$ be the closure of the union of all of the $N(\theta), \theta \in[0, \pi)$. Notice that $N$ is not a 3 -ball (it is a 3 -ball with holes), because $N(\theta)$ is not a disc for $\theta$-values close to $\theta=0$. Choose $\epsilon>0$ so that $N(\theta)$ is a disc for $\theta \in[\epsilon, \pi-\epsilon]$.
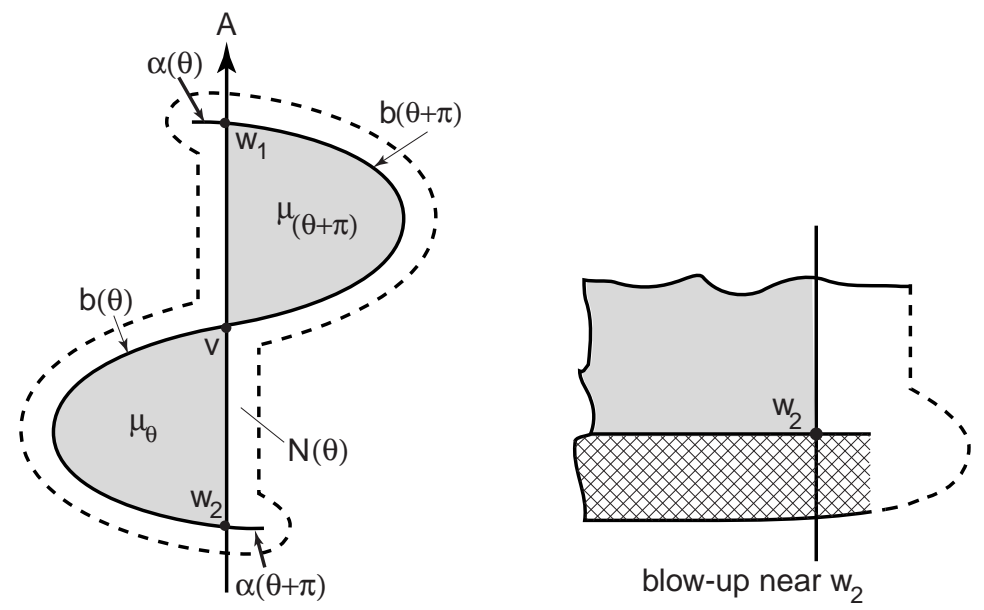

Figure 2.18

We can now describe precisely the exchange move which was indicated earlier in Figure 2.15. See the top left picture in Figure 2.19, which is 
intended to correspond to the left picture in Figure 2.18, at some $\theta \in(\epsilon, \pi-$ $\epsilon)$. The plane of the paper is divided by $A$ into two half-planes, and we will assume the left one to be $H_{\theta}$ and the right one to be $H_{\theta+\pi}$. We want to describe an isotopic deformation of $\mathcal{S} \cup \mathcal{L}$, and shall do so by the series of pictures in Figure 2.19, all of which correspond to the same fixed value of $\theta \in(\epsilon, \pi-\epsilon)$.

Choose a subdisc $d$ of $\mu_{\theta+\pi}$ such that $d \cap L=\mu_{\theta+\pi} \cap L$ and also $d \cap \mathcal{S}=$ $\mu_{\theta+\pi} \cap \mathcal{S} \backslash\lfloor(\theta+\pi)$. Our isotopy is to be supported in $N(\theta)$ and is to be the identity on $\mu_{\theta}$ and on $\mu_{\theta+\pi}$ minus a neighborhood of $d$ on $\mu_{\theta+\pi}$. The isotopy pushes the disc $d$ (and the points of $L$ and $\mathcal{S}$ which meet it) across $A$ and then down and eventually into the cross-hatched area below $w_{2}$ which was illustrated in the blow-up in Figure 2.18. The isotopy is to be defined on pairs of fibers, and is to be defined so that it varies continuously as we vary $\theta$. At the end of the isotopy the $\operatorname{link} L$ is to encircle the axis below $w_{2}$ instead of between $w_{1}$ and $v$. Also, each sheet of $\mathcal{S}$ which intersected $A$ between $w_{1}$ and $v$ is to intersect $A$ the same number of times below $w_{2}$. (One can think of this change as accomplished by "putting your hand into your pocket and emptying it".)

The only remaining problem is to ask what happens in the $\theta$-interval $[0, \epsilon]$ ? Let $u$ be the isotopy parameter. The first thing to notice is that when $u$ is in the interval $[.25, .75]$ the isotopy is supported in the left half-plane. We may then assume that the deformed discs $d_{u}, u \in[.25, .75]$, have empty intersection with $H_{\theta+\pi}$ if $\theta$ is not in the interval $[\epsilon, \pi-\epsilon]$. The second thing to notice is that when $u \in[0, .25]$ and $u \in[.75,1]$ the isotopy is supported in a neighborhood of the axis $A$. Since that neighborhood can be chosen to be disjoint from a neighborhood of the singular points, it follows that Figure 2.19 actually tells us everything we need to describe the isotopy of $\mathcal{S} \cup \mathcal{L}$ completely.

At the end of the isotopy, we may reposition $\mathcal{S}$ so that its decomposition is exactly as it was before the change. There has, however, been a change in the order of the points of $A \cap \mathcal{S}$ on $A$. After the change $b\left(\theta_{7}\right)$ and possibly some leaves parallel to it will become inessential. We can therefore modify the surface by an additional isotopy to a new surface $\mathcal{S}^{\prime}$, by eliminating all inessential $b$-arcs and any resulting unwanted $c$-circles, in the manner described in the proofs of Lemmas 1.2, 1.3 and 1.4. Thus, the decomposition of $\mathcal{S}^{\prime}$ has at least two fewer vertices and at least two fewer regions than the decomposition of $\mathcal{S}$. $\|$ 


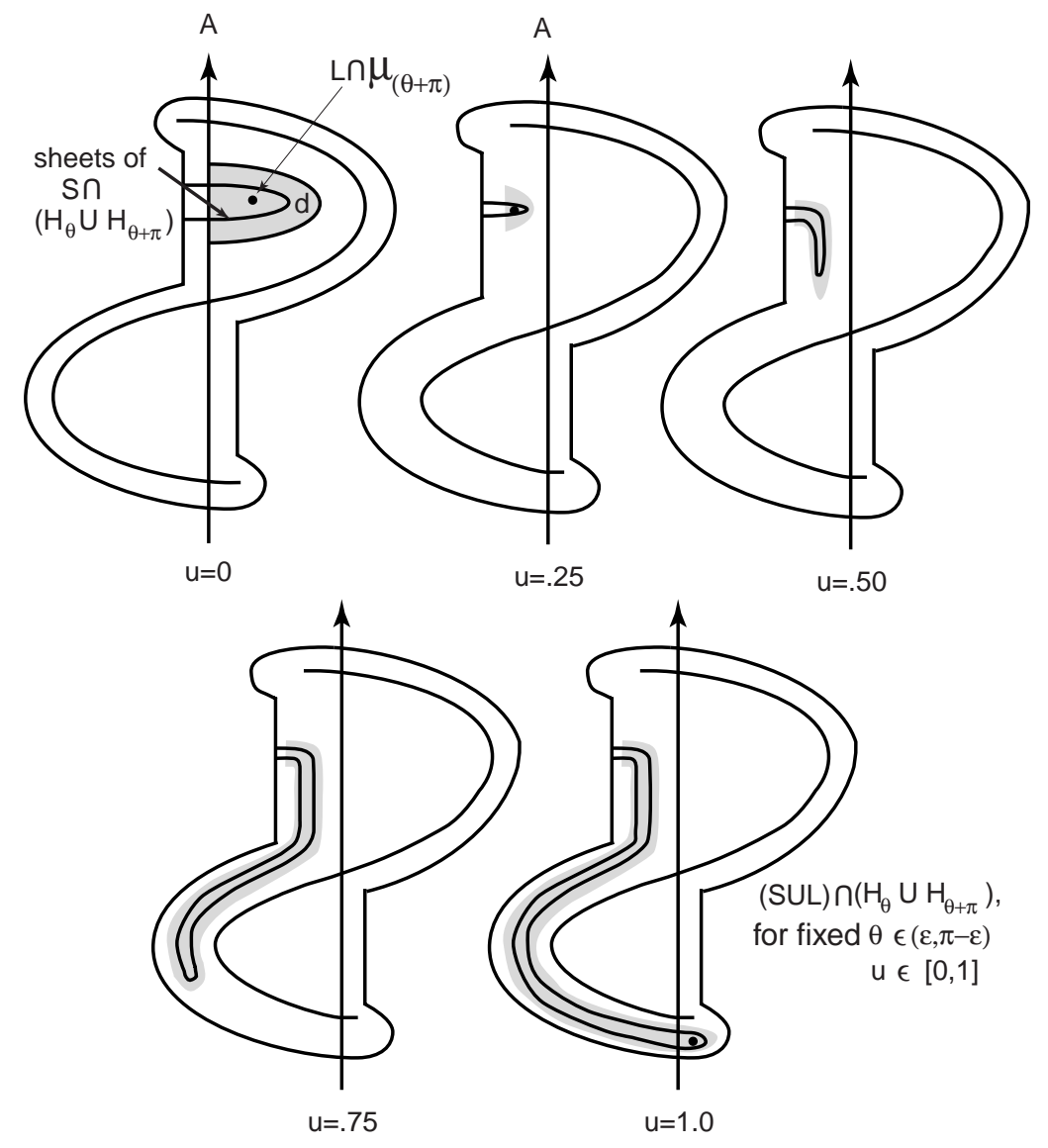

Figure 2.19

\section{$\S$ 3. GLOBAL TOPOLOGY OF THE TILING OF $\mathcal{F}$.}

In the previous section we discussed particular ways in which we could make local changes in the tiling of $\mathcal{F}$ and in the decomposition of $\mathcal{C}$. In this section we discuss the 'global topology' of the tiling of $\mathcal{F}$. Clues about the global topology of $\mathcal{F}$ may be found in four graphs defined by the tiling, which we will introduce shortly.

The following observation will be useful in several places in this section:

Observation $\star$ : If we change the orientation on $L$ then every \pm vertex becomes a $\mp$ vertex. If we change the orientation on the fibers of $H$ then every \pm singular point becomes a $\mp$ point. In this way certain proofs which 
assume a particular choice of sign $\epsilon$ for a vertex and $\operatorname{sign} \delta$ for a singular point may be adapted to apply to the four possible choices of $(\epsilon, \delta)$.

Our initial goal is to locate the places on the tiling of $\mathcal{F}$ where the changes of Section 2 can be made. We begin with some new definitions. Let $v$ be a vertex. A vertex $v_{i}$ in the tiling of $\mathcal{F}$ is said to be near $v$ if there is a $b$-arc $\beta_{i}$ in the foliation of $\mathcal{F}$ with $\partial \beta_{i}=v \cup v_{i}$. A singular point $s_{j}$ in the tiling of $\mathcal{F}$ is said to be near $v$ if $s_{j}$ lies on a singular leaf $l_{j}$ which ends at $v$. Let $\operatorname{star}(v)=\left\{v_{i}, s_{j} \mid v_{i}, s_{j}\right.$ are near $\left.v\right\}$.

See Figure 3.1 for two examples. In the first $\operatorname{star}(v)$ has arbitrarily many vertices, but in the second there are only two vertices in $\operatorname{star}(v)$. A vertex $v$ in the tiling of $\mathcal{F}$ is said to be an interior vertex if it is not an endpoint of an $a$-arc.

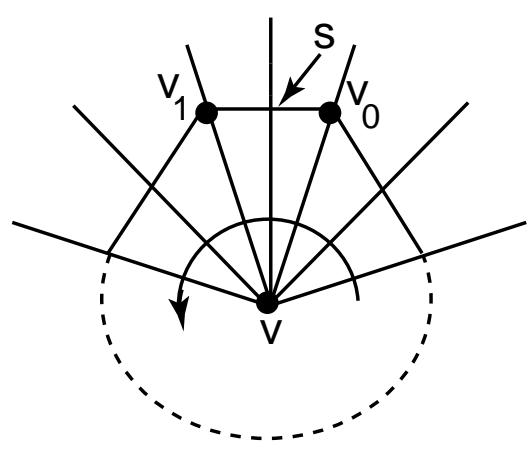

(a)

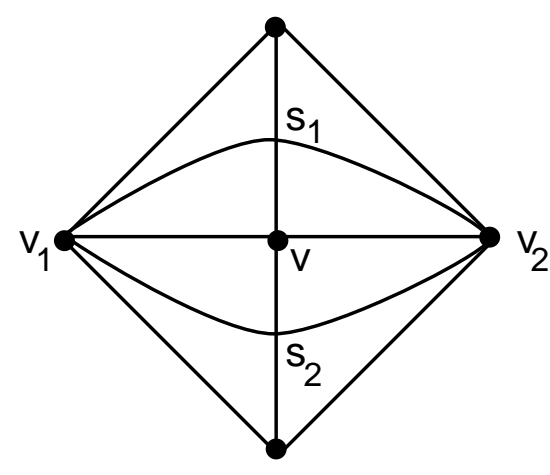

(b)

Figure 3.1

Our first result in this section was explained to us by Menasco. He says that it is implicit in $\mathbf{B e}$. However, it is very difficult to pinpoint it there and at this writing Menasco's version of it is unpublished, so it appears here for the first time.

Lemma 3.1 Let $v$ be an interior vertex. Then star $(v)$ contains both positive and negative singular points.

Proof of Lemma 3.1. By Observation $\star$ we may assume without loss of generality that $v$ is a positive vertex. Let $v_{1}, v_{2}, \ldots, v_{r}$ be the vertices in $\operatorname{star}(v)$, ordered so that their cyclic order on the oriented braid axis $A$ is $v, v_{1}, v_{2}, \ldots, v_{r}$ (see Figure 3.2(a)). Thus there are $r$ vertices in $\operatorname{star}(v)$, also 
$v_{1}$ is the first vertex in $\operatorname{star}(v)$ which is encountered if one starts at $v$ and travels along the oriented braid axis $A$ in the positive direction.

The vertices $v_{1}, \ldots, v_{r}$ also have a second order, in the flow around $v$. Traveling around $v$ counterclockwise, in the direction of increasing polar angle, let $v_{0} \in\left\{v_{1}, \ldots, v_{r}\right\}$ be the vertex which occurs just before $v_{1}$, and let $s$ be the singular point in $\operatorname{star}(v)$ which is between $v_{0}$ and $v_{1}$ (see Figure $3.2(\mathrm{~b}))$.

Choose a non-singular fiber $H_{\theta_{1}}$ which contains a $b$-arc $\beta_{1}$ joining $v$ to $v_{1}$, as in Figure $3.2(\mathrm{c})$. The arc $\beta_{1}$ divides $H_{\theta_{1}}$ into into two half-discs, which we call $H_{\theta_{1}}^{+}$and $H_{\theta_{1}}^{-}$, where the signs are chosen so that $H_{\theta_{1}}^{+}$(resp. $H_{\theta_{1}}^{-}$) is the side of $H_{\theta_{1}}$ split along $\beta_{1}$ which meets the + (resp. - ) side of $\mathcal{F}$. The fact that the order of the vertices on $A$ is $v, v_{1}, \ldots, v_{r}$ then implies that the vertices $v_{2}, \ldots, v_{r}$ are all on the $\partial H_{\theta_{1}}^{-}$part of $A$.

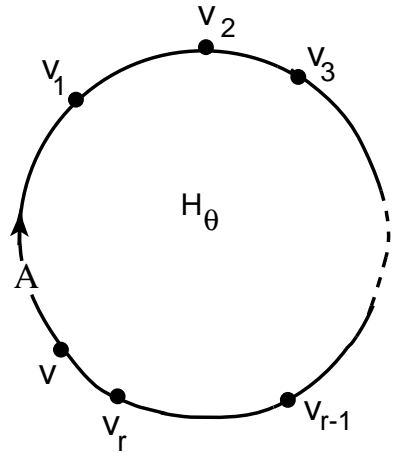

(a)

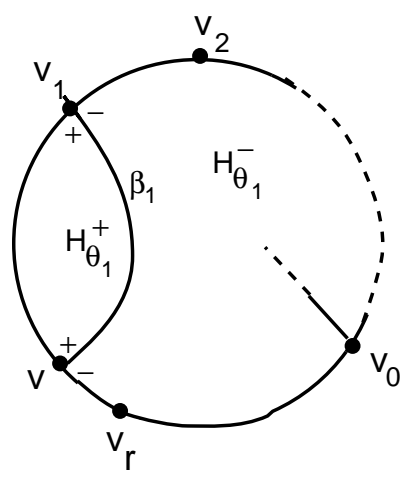

(c) the fiber $\mathrm{H}_{\theta_{1}}$

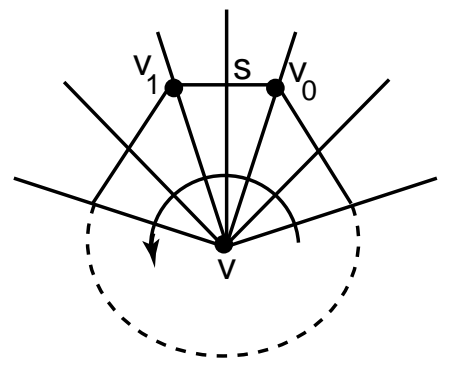

(b)

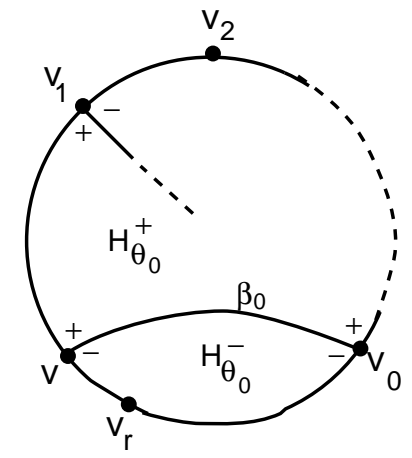

(d) the fiber $\mathrm{H}_{\theta_{0}}$

Figure 3.2 
Notice that the sign of a singularity has natural meaning when it is examined in a sequence of fibers of $H$. The surface $\mathcal{F}$ is oriented, so that when we view a component of $\mathcal{F} \cap \mathcal{H}_{\theta}$ on $H_{\theta}$ it will have a well-defined positive and negative side. See Figure 1.11 for a series of pictures which show the leaves of $\mathcal{F} \cap \mathcal{H}_{\theta}$ just before, at the instant of, and just after a positive singularity. When the singularity is positive (resp. negative) the negative (resp. positive) sides of $\mathcal{F} \cap \mathcal{H}_{\theta}$ approach one-another just before the singularity and then split apart in a new way. This means that, following the flow around $v$, the first singularity occurring after $\beta_{1}$ must be positive. Otherwise, $\beta_{1}$ would surger with a $b$-arc having an endpoint on $\partial H_{\theta_{1}}^{+}$, but since $v_{2}, \ldots, v_{r}$ are on $\partial H_{\theta_{1}}^{-}$, this is impossible.

Recall how the vertex $v_{0}$ and the singular point $s$ were chosen. In particular, recall that $s$ is the singular point in $\operatorname{star}(v)$ which occurs between $v_{0}$ and $v_{1}$. Thus, if $\beta_{1}$ and $\beta_{0}$ are the b-arcs joining $v$ to $v_{1}$ and $v_{0}$, respectively, then $\beta_{0}, s, \beta_{1}$ have that order in the fibration. When viewed on a sequence of fibers of $H$, we see that the singularity at $s$ results in the creation of the $b$-arc $\beta_{1}$, and that just before this singularity occurs there is a non-singular fiber $H_{\theta_{0}}$ containing the $b$-arc $\beta_{0}$ (see Figures $3.2(\mathrm{c})$ and (d)). The fact that $v$ is positive forces $v_{0}$ to be negative, because $v$ and $v_{0}$ are the two endpoints of a $b$-arc. This means that the oriented axis $A$ intersects the positive side of $\mathcal{F}$ first at $v_{0}$. It follows that $v_{1}$ must be on the negative side of $H_{\theta_{0}}$ split along $\beta_{0}$. But then, the singularity at $s$ must be negative. $\|$

Let $\vec{x}=x, \ldots, x$ where $x$ is either a sign \pm or a $b$-arc.

Lemma 3.2 After some number of changes in foliation, exchange moves and isotopies in the complement of the axis, it may be assumed that no interior vertex has sign $(\overrightarrow{+}, \overrightarrow{-})$.

Proof of Lemma 3.2. Let $V(\mathcal{F})$ be the set of all vertices in the tiling of $\mathcal{F}$. Recall that $V$ is the cardinality of $V(\mathcal{F})$. Suppose that $v \in V(\mathcal{F})$ is an interior vertex with $\operatorname{sign}(\overrightarrow{+}, \overrightarrow{-})$. Since $v$ is an interior vertex, it has type $(\vec{b})$. By Theorem 2.1 we may assume, after changes in foliation which push off adjacent tiles of like sign, that $v$ has valence 2 , type $(b, b)$ and sign $(+,-)$. The vertex $v$ is still an interior vertex, so Theorem 2.2, part (2.2.2) applies. The link $L$ admits an exchange move, and after the exchange move we may eliminate an inessential $b$ arc which has $v$ as its endpoint, by an isotopy of $\mathcal{F}$ which is supported in a neighborhood of the tiles which meet $v$. Since this process eliminates $v$ and creates no new vertices, $V$ is reduced. If there is again an interior vertex $v$ with $\operatorname{sign}(\overrightarrow{+}, \overrightarrow{-})$ the argument may be repeated 
and, since $V$ is reduced each time, a finite number of repetitions will yield a tiling in which no interior vertex has $\operatorname{sign}(\overrightarrow{+}, \overrightarrow{-})$. $\|$

Lemma 3.3 After some number of changes in foliation, exchange moves, isotopies in the complement of the axis, and destabilizations along vertices of type (a), it may be assumed that if a vertex $v$ has type $(\vec{b}, a, \vec{b})$, then there are singularities of opposite sign in star $(v)$.

Proof of Lemma 3.3. Suppose there is such a vertex $v$ in the set $V(\mathcal{F})$ of all vertices in the tiling of $\mathcal{F}$. By Theorem 2.1 and Remark 2.1 we may assume, after changes in foliation which push off tiles adjacent at a $b$-arc of like sign, that $v$ has type $(a)$. A destabilization move via the vertex $v$ then eliminates it. Since this process creates no new vertices, $V$ is reduced. If there is again a vertex as described in Lemma 3.3 the argument may be repeated and, since $V$ is reduced each time, we may assume that Lemma 3.3 holds after a finite number of repetitions. $\|$

Lemma 3.4 After some number of changes in foliation, exchange moves, isotopies in the complement of the axis, and destabilizations along vertices of type (a), it may be assumed that no vertex $v$ has type $(\vec{b}, a, \vec{b})$ and sign $(\overrightarrow{+}, \overrightarrow{-})$, where one of the changes from + to - corresponds to singularities in star $(v)$ of opposite sign in ab-tiles adjacent at the boundary a-arc which meets $v$.

Proof of Lemma 3.4. Suppose there is such a vertex $v$ in the set $V(\mathcal{F})$ of all vertices in the tiling of $\mathcal{F}$. By Theorem 2.1 we may assume, after changes in foliation which push off tiles adjacent at a $b$-arc of like sign, that $v$ has valence 2 , type $(a, b)$ and sign $(+,-)$. By Theorem 2.2, part (2.2.1), the link $L$ admits an exchange move and an isotopy of $\mathcal{F}$ eliminates $v$. Since this process creates no new vertices, $V$ is reduced. If there is again a vertex as described in Lemma 3.4 the argument may be repeated and, since $V$ is reduced each time, we may assume that Lemma 3.4 holds after a finite number of repetitions. $\|$

To continue our work, we introduce four graphs, denoted $G_{++}, G_{+-}$, $G_{-+}$and $G_{--}$, whose edges are singular leaves in the positive and negative tiles in the tiling of $\mathcal{F}$. See Figure 3.3. These graphs were first considered by Bennequin in $[\mathbf{B e}$, who was interested in them in the special case when $\mathcal{F}$ is a disc. They are in some ways dual to the tilings, and they encode topological properties of the surface in new ways. They were used by Menasco in [M] in his study of unknotting numbers of knots and by Fung in his thesis [Fu]. 
No doubt they have other applications too. All four graphs are subsets of
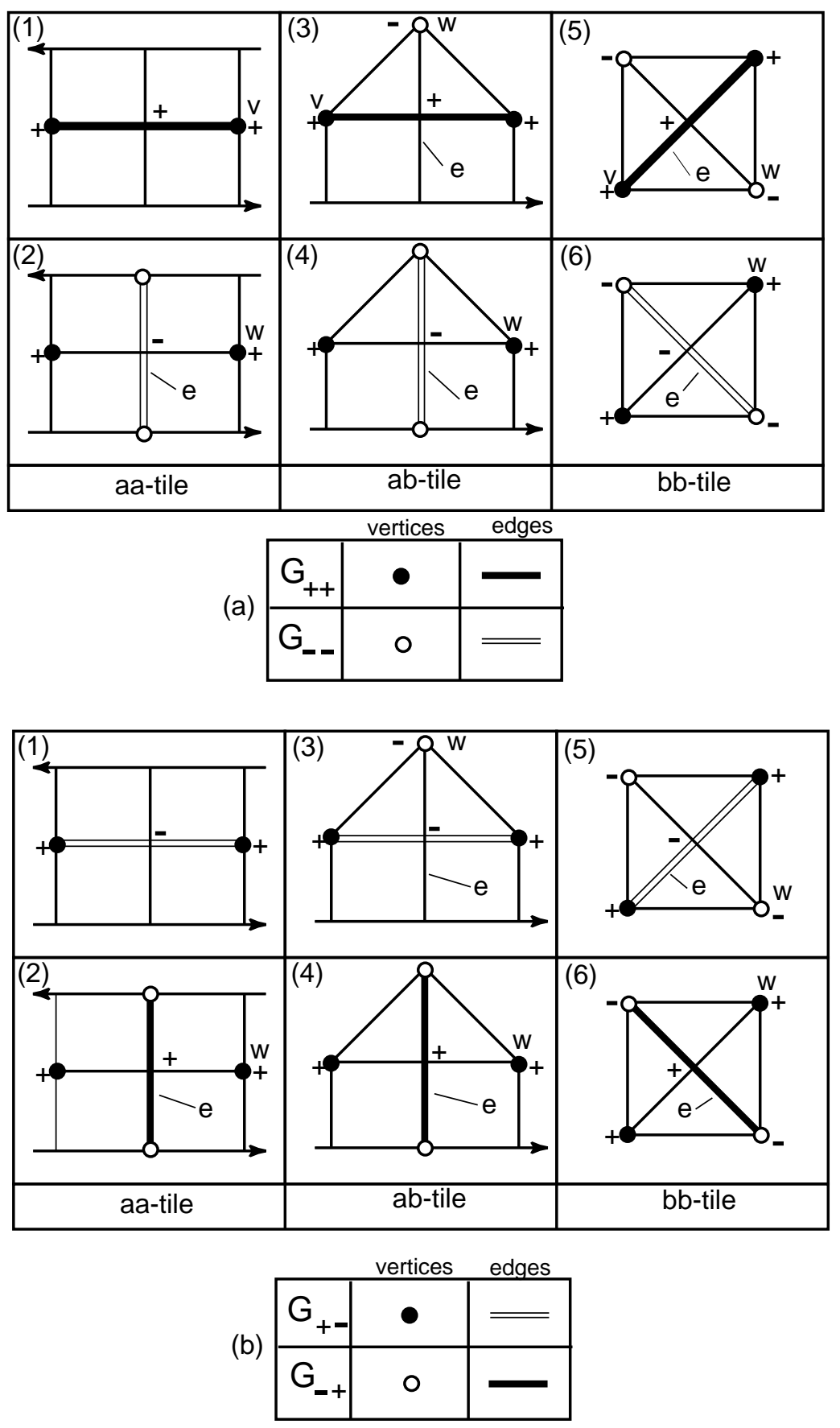

Figure 3.3 
the singular leaves in the foliation of $\mathcal{F}$. The graph $G_{\epsilon, \delta}$ (consult Figure 3.3) passes through only vertices of $\operatorname{sign} \epsilon$ and singular points of sign $\delta$, where $\epsilon$ and $\delta$ are fixed signs, each \pm . However, they have rather different structures and pick up different aspects of the combinatorics of the tiling, because the three tile types in a spanning surface are quite different as regards their positive and negative vertices. Recall that the sign of a tile is the sign of the unique singular point in that tile.

See Figure 3.3 for illustrations of the edges and vertices in $G_{++}, G_{+-}$, $G_{-+}$and $G_{--}$. The black vertices indicate vertices of $G_{+, \delta}$ and the circled vertices indicate vertices of $G_{-, \delta}$. The thick black lines indicate edges of $G_{\epsilon,+}$, and the double lines indicate edges of $G_{\epsilon,-}$. Notice that the graphs $G+,-$ and $G_{-,+}$are obtained from $G_{+,+}$and $G_{-,-}$by reversing the orientation of the fibers of $H$.

Definition (See Figure 3.3):

- The graph $G_{+, \delta}$ has as its edges those subarcs of singular leaves which join the two + vertices in a $a a, a b$ or $b b$ tile of sign $\delta$. The vertices of $G_{+, \delta}$ are the endpoints of these edges, together with all + vertices in the tiling of $\mathcal{F}$ which are not adjacent to any singular point of sign $\delta$.

- The graph $G_{-, \delta}$ has as its edges those subarcs of singular leaves which:

- join the two negative vertices in a $b b$ tile of sign $\delta$.

- join the negative vertex in an $a b$ tile of sign $\delta$ to $\partial \mathcal{F}$.

- join $\partial \mathcal{F}$ to $\partial \mathcal{F}$ in an $a a$ tile of sign $\delta$.

The vertices of $G_{-, \delta}$ are the endpoints of these edges, together with negative vertices in the tiling of $\mathcal{F}$ which are not adjacent to any singular point of sign $\delta$.

Remark 3.1 If one thinks of $\partial \mathcal{F}$ as acting "like a negative vertex" (see Remark 1.1) then the definition of $G_{-, \delta}$ will be seen to be analogous to that of $G_{+, \delta}$.

Remark 3.2 Note that there are vertices of $G_{\epsilon, \delta}$ that are not vertices of tiles in the tiling of $\mathcal{F}$. To avoid confusion we will refer to the former as vertices of $G_{\epsilon, \delta}$ and the later as vertices in the tiling of $\mathcal{F}$.

Lemma $3.5[\mathbf{M}]$. The graphs $G_{\epsilon, \delta}$ have the following properties:

1. $G_{\epsilon, \delta} \cap G_{-\epsilon,-\delta}=\emptyset$. 
2. Every singular point in the foliation of $\mathcal{F}$ is in $G_{++}$or $G_{--}$(and so also in $G_{+-}$or $G_{-+}$).

3. Every vertex in the tiling of $\mathcal{F}$ is a vertex of $G_{++}$or $G_{--}$( and so also in $G_{+-}$or $G_{-+}$).

Proof of Lemma 3.5: It suffices to prove the assertions for $G_{+,+}$and $G_{-,-}$, since the other two cases follow by reversing the orientation of fibers of $H$.

1. The vertices and singular points of $G_{++}$and $G_{--}$have distinct signs. Since each edge of $G_{++}$and $G_{--}$contains a singular point, the only possibility is that $G_{++} \cap G_{--}=\emptyset$.

2. Each singular point $s$ in the foliation of $\mathcal{F}$ belongs to either an $a a$ or $a b$ or $b b$-tile, and a check of the possibilities shows that $s$ belongs to an edge of $G_{++}$if the sign of $s$ is positive and an edge of $G_{--}$if its sign is negative.

3. This follows from (2). ॥

A vertex of $G_{\epsilon, \delta}$ is isolated if it is not the endpoint of any edge of $G_{\epsilon, \delta}$.

Lemma 3.6. No graph $G_{\epsilon, \delta}$ contains an isolated vertex which is an interior vertex in the tiling of $\mathcal{F}$.

Proof of Lemma 3.6 Suppose that for some choice of $(\epsilon, \delta)$ the vertex $v$ is an isolated interior vertex in $G_{\epsilon, \delta}$. Since $v$ is an isolated vertex, every singular point in $\operatorname{star}(v)$ must have sign $-\delta$. However this contradicts Lemma 3.1. II

An endpoint of $G_{\epsilon, \delta}$ is a vertex of $G_{\epsilon, \delta}$ which is the endpoint of exactly one edge of $G_{\epsilon, \delta}$.

Lemma 3.7 After some number of changes in foliation, exchange moves and isotopies in the complement of the axis, it may be assumed that no graph $G_{\epsilon, \delta}$ has an endpoint vertex which is an interior vertex in the tiling of $\mathcal{F}$.

Proof of Lemma 3.7. Suppose $v$ is an endpoint vertex of $G_{\epsilon, \delta}$ and an interior vertex in the tiling of $\mathcal{F}$, for some fixed $\epsilon$ and $\delta$. Since $v$ is an interior vertex in the tiling of $\mathcal{F}$ we may assume by Lemma 3.2, after some number of changes in foliation, exchange moves and isotopies in the complement of the axis, that $v$ does not have $\operatorname{sign}(\overrightarrow{+}, \overrightarrow{-})$. But, since $v$ is an endpoint vertex of $G_{\epsilon, \delta}$, there is exactly one singularity in $\operatorname{star}(v)$ with sign $\delta$ and all others have sign $-\delta$. Thus, $v$ has sign $(\delta, \overrightarrow{-} \delta)$, a contradiction. $\|$ 
Lemma 3.8 After some number of changes in foliation, exchange moves, isotopies in the complement of the axis, we may assume that:

(i) no closed loop in $G_{\epsilon, \delta}$ bounds a disk on $\mathcal{F}$;

(ii) no closed loop which is the union of an edgepath in $G_{\epsilon,+}$ and an edgepath in $G_{\epsilon,-}$ bounds a disk on $\mathcal{F}$;

(iii) after some number of destabilizations along vertices of type (a), no closed loop which is a union of edges in $G_{-, \delta}$ and one subarc of $L$ bounds a disk on $\mathcal{F}$ and

(iv) after some number of destabilizations along vertices of type (a), no closed loop which is the union of an edgepath in $G_{-,+}$, an edgepath in $G_{-,-}$and a subarc of $L$ bounds a disk on $\mathcal{F}$;

Proof of Lemma 3.8. By Lemmas 3.2, 3.6 and 3.7, we may assume, after some number of changes in foliation, exchange moves, isotopies in the complement of the axis, that no graph $G_{\epsilon, \delta}$ contains an interior vertex in the tiling of $\mathcal{F}$ which has sign $(\overrightarrow{+}, \overrightarrow{-})$, and no graph $G_{\epsilon, \delta}$ contains an isolated or endpoint vertex that is also an interior vertex in the tiling of $\mathcal{F}$.

Suppose (i) or (ii) is false. Then there is a closed loop $c$ of the type described in (i) or (ii) which bounds a disk $D$ on $\mathcal{F}$. Choose $c$ to be minimal in the sense that there is no closed loop as described in (i) or (ii) which bounds a proper subdisk of $D$. The loop $c$ is either in $G_{\epsilon, \delta}$ for some choice of $\epsilon, \delta$, or is equal to the union of an edgepath in $G_{\epsilon,+}$ and an edgepath in $G_{\epsilon,-}$, for some choice of $\epsilon$. Therefore any edge $e$ of $c$ is in $G_{\epsilon, \mu}$, where $\mu=1$ or $\mu=-1$. Thus, since $D \cap L=\emptyset, e$ is in a tile which contains a vertex $w$ in $D$ of parity $-\epsilon$ as shown in (3),(5) or (6) of Figures 3.3(a)(b), depending on the signs of $\epsilon, \mu$. Since $w$ has parity $-\epsilon, w$ is in $\operatorname{Int}(D)$.

We have just shown that $G_{-\epsilon, \mu}$ has non-empty intersection with $\operatorname{Int}(D)$ for $\mu- \pm 1$, but what about $G_{\epsilon, \mu}$ ? Note that $G_{\epsilon, \mu} \cap \operatorname{Int}(D)$ contains only tree components with endpoints on $c$, since otherwise either $c$ is not minimal or $G_{\epsilon, \mu}$ contains an endpoint or isolated vertex which is an interior vertex in the tiling of $\mathcal{F}$. Suppose $T$ is such a tree component. Note that each endpoint of $T$ must be a vertex of $c$. For, if some endpoint of $T$ is a singularity of $c$, that singularity must be in a tile having three vertices of the same parity $\epsilon$. But since no tile type has more than two vertices of the same parity, we conclude that $T$ has an edgepath $\alpha$ with endpoints equal to vertices of c. The edgepath $\alpha$ separates $D$ into two subdisks. But each subdisk has boundary in $G_{\epsilon, \mu}$ or has boundary equal to the union of an edgepath in 
$G_{\epsilon,+}$ and an edgepath in $G_{\epsilon,-}$, contradicting the minimality of $c$. We may therefore assume that $G_{\epsilon, \mu} \cap \operatorname{Int}(D)=\emptyset$ for $\mu= \pm 1$.

By Lemma $3.5(2)$, no singularities in the foliation of $\mathcal{F}$ are contained in $\operatorname{Int}(D)$. But then all singularities in $\operatorname{star}(w)$ are on $c$ and each vertex of $c$ is connected to $w$ by a $b$-arc. Thus, $w$ has type $(\vec{b})$, and $\operatorname{sign}(\vec{\delta})$, if $c$ is in $G_{\epsilon, \delta}$ or sign $(\overrightarrow{+}, \overrightarrow{-})$ if $c$ is the union of an edgepath in $G_{\epsilon,+}$ and $G_{\epsilon,-}$. The first case contradicts Lemma 3.1 and the second contradicts Lemma 3.2. This proves (i) and (ii).

We now assume that, after some number of destabilizations along vertices of type (a), that Lemmas 3.3 and 3.4 hold in addition to the Lemmas 3.2, 3.6 and 3.7. Suppose that (iii) or (iv) is false. Then there is a closed loop $c$ of the type described in (iii) or (iv) which bounds a disk $D$ on $\mathcal{F}$. Choose $c$ to be minimal in the sense that there is no closed loop as described in (iii) or (iv) which bounds a proper subdisk of $D$. Note that every edge $e$ of $c$ is in $G_{-, \delta}$ for some choice of $\delta= \pm 1$, so $e$ is as shown in one of Figures (2)(4)(6) of 3.3 (a) (respectively $3.3(\mathrm{~b}))$ if $\delta=-$ (respectively $\delta=+$ ). Thus, there is a positive vertex $w$ in $D$. Since every vertex of $c$ is negative, $w$ is in $\operatorname{Int}(D)$. Note that $G_{-, \delta} \cap \operatorname{Int}(D)=\emptyset$ for $\delta= \pm 1$. For, suppose otherwise. By (i), there are no closed loops, and since every vertex in $G_{-, \delta} \cap \operatorname{Int}(D)$ is an interior vertex in the tiling of $\mathcal{F}, G_{-, \delta} \cap \operatorname{Int}(D)$ contains no endpoint or isolated vertices. Thus, $G_{-, \delta} \cap \operatorname{Int}(D)$ consists of tree components with endpoints on $c$, and $T$ contains an edgepath $\alpha$ with endpoints on $c$. No endpoint of $\alpha$ can be a singularity of $c$, since otherwise there is a tile with three negative vertices. Thus, the endpoints of $\alpha$ may be points on $L$ or vertices of $c$. It is easy to check that all possible cases contradict (i), (ii) or minimality of $c$, and we conclude that $G_{-, \delta} \cap \operatorname{Int}(D)=\emptyset$ for $\delta= \pm 1$. By Lemma 3.5, we may assume that $\operatorname{Int}(D)$ contains no singularities in the foliation of $\mathcal{F}$. Thus, all singularities of $\operatorname{star}(w)$ are on $c$ and each vertex of $c$ is connected to $w$ by a $b$-arc. Thus, $w$ has type $(\vec{b}, a, \vec{b})$. If $c$ is a loop of the type described in (iii), then all singularities in $\operatorname{star}(w)$ have the same sign, contradicting Lemma 3.3. If $c$ is a loop of the type described in (iv), then $w$ has $\operatorname{sign}(\overrightarrow{+}, \overrightarrow{-})$, where one of the changes from + to - corresponds to singularities in $\operatorname{star}(w)$ of opposite sign in $a b$-tiles adjacent at the boundary $a$-arc which meets $w$. But this contradicts Lemma 3.4 and concludes the proof of Lemma 3.8. \|

Theorem 3.1. After some number of changes in foliation, exchange moves and isotopies in the complement of the axis, it may be assumed that each of the following holds for all four graphs at once:

(i) $G_{\epsilon, \delta}$ has no interior endpoint vertices. 
(ii) $G_{\epsilon, \delta}$ has no interior isolated vertices.

(iii) $G_{\epsilon, \delta}$ contains no closed loop bounding a disk on $\mathcal{F}$.

(iv) there is no closed loop equal to the union of an edgepath in $G_{\epsilon,+}$ and an edgepath in $G_{\epsilon,-}$ which bounds a disk on $\mathcal{F}$.

(v) after some number of destabilizations at vertices of type (a), there is no closed loop equal to the union of an edge-path in $G_{-, \delta}$ and a subarc of $L$ which bounds a disk on $\mathcal{F}$.

(vi) after some number of deletions of trivial loops of $L$, there is no closed loop equal to the union of an edge-path in $G_{-,+}$, an edge-path in $G_{-,-}$ and a subarc of $L$ which bounds a disk on $\mathcal{F}$.

Proof of Theorem 3.1. This follows from Lemmas 3.6, 3.7 and 3.8. ॥

\section{$\S$ 4. APPLICATIONS AND OPEN PROBLEMS.}

In this section we prove three results (Theorems 4.1, 4.2 and 4.3) which illustrate how the methods which were described in Sections 1-3 may be used to solve problems about surfaces which have a special position with regard to a link. We also pose some interesting open problems which should yield through the use of the techniques in this paper. With regard to the theorems: Theorem 4.1 is from [BM 6]. To the best of our knowledge Theorem 4.2 has not been written down anywhere, although it is implicit in the work of Bennequin and Birman-Menasco. Theorem 4.3 is the main result from BM 5, but the proof which we give here is new and is simpler than the original proof, because we use the graphs $G_{\epsilon, \delta}$, which were not known when [BM 5] was written.

Our first application relates to Theorem 1.2, in the special case when $\mathcal{S}=\mathcal{F}$. Let us suppose that $L$ is a closed $n$-braid with respect to the braid axis $A$ and that $\mathcal{F}$ is decomposed into tiles of types $a a, a b$ and $b b$. Suppose that there are $t$ singularities in the foliation of $\mathcal{F}$, that $A$ intersects $\mathcal{F}$ in $k$ points, and that there are $r$ points on $L$ which are the endpoints of singular leaves in the tiling. Assume that the singularities $s_{1}, s_{2}, \ldots, s_{t}$ have been numbered in the cyclic order in which they occur in the fibration, the vertices have been numbered $v_{1}, v_{2}, \ldots, v_{k}$ in the natural order in which they occur on $A$, and the endpoints of the singular leaves which are on $L$ have been numbered $p_{1}, p_{2}, \ldots, p_{r}$ in their natural cyclic order on $L$.

Theorem 4.1. The following combinatorial data for the tiling of $\mathcal{F}$ determines the embedding of $L$ (respectively $\mathcal{F}$ ): 
(1) A listing of the number of regions of type aa and $a b$ (respectively $a a, a b$ and $b b)$.

(2) For each region, its sign and an identification of its vertices among the vertices $v_{i}$ on $A$.

(3) For each region of type aa and ab, an identification of its L-endpoints among the points $p_{j}$ on $L$.

(4) For each region, the order of the singularity it contains in the cycle $s_{1}, \ldots, s_{t}$.

Proof of Theorem 4.1. In the proof of Lemma 1.6 we showed how each $a b$-tile has a canonical embedding in 3-space, up to an isotopy which is supported on the axis $A$ and the singular fiber $H_{\theta}$ associated to the tile. The embedding is determined by the data of type (2) above. A small modification of the argument shows that each $a a$ and $b b$-tile also has a similar canonical embedding. See Figure 1.13 of $\S 1$ for illustrations of the canonical embeddings of the three tile types. The embedding of $\mathcal{F}$ is determined by specifying how the boundary components of the embedded regions are identified. Identifications of boundary components which are $a$-arcs are specified by the data of type (3). Two $b$-arc boundary components of regions $R_{1}$ and $R_{2}$ are identified if they have two common vertices and the singularities belonging to the regions $R_{1}$ and $R_{2}$ occur consecutively in the fibration. Thus, the data of type (2) and (4) determines how the boundary components of regions which are $b$-arcs are identified. The embedding of $\mathcal{F}$ is therefore determined by the data of types (1), (2), (3) and (4). If we are only interested in the embedding of $L$, then we do not need to include the information for $b b$-tiles because they do not meet $L$. $\|$

Problem 4.1. A word in the standard elementary braid generators $\sigma_{1}, \ldots, \sigma_{n-1}$ of the braid group $B_{n}$ and their inverses gives a description of a closed braid by a finite set of combinatorial data. Theorem 4.1 gives an alternative description, which in principle contains more information. In principle, the new data implies the old data, however we do not at this time know any direct algorithm which allows us to pass from the combinatorial data of Theorem 4.1 to a braid word which describes $L$, although it is clear that such an algorithm must exist. It would be interesting to have such an algorithm. The key to the difficulty seems to be to understand the way in which $a b$-tiles affect the braid word.

Remark 4.1: Theorem 4.1 generalizes to the case of closed surfaces, if one includes additional data on the ways in which regions of type $b b, b c$ and $c c$ 
are pasted together along their boundaries.

Problem 4.2. We showed, in Theorem 4.1, that the tiling (plus some additional combinatorial data) determines the embedded surface. On the other hand, it is fairly simple to construct a tiling of a surface of genus $g$ with boundary which is not geometrically realizable by a spanning surface for a closed braid. We pose, as an open problem, to determine necessary and sufficient conditions such that an abstract decomposition of a surface into foliated regions, as described in Section 1, is realized by an embedded surface. There are obvious variations on this: e.g. when is the surface incompressible etc.

Recall that a Markov surface is, by definition, a surface of minimal genus with boundary a given closed braid. The closed braid will not, in general, have minimal braid index. A special Markov surface is a Markov surface which is tiled entirely by aa-tiles. Our next result shows that every link is the boundary of a special Markov surface.

Theorem 4.2 Every link type $\mathcal{L}$ has a closed braid representative $L^{*}$ which is is the boundary of a special Markov surface $\mathcal{F}^{*}$.

Proof of Theorem 4.2. Choose a Markov surface $\mathcal{F}$ for any closed braid representative $L$ of $\mathcal{L}$. If the tiling of $\mathcal{F}$ contains $a b$-tiles, we may stabilize along them, as in $\S 2.2 .1$. Each stabilization move deletes a negative vertex at the expense of adding a trivial loop. Thus, although new $a b$-tiles may be created from $b b$-tiles during a stabilization move, finitely many stabilizations will eliminate all of the $a b$-tiles, since $\mathcal{F}$ has finitely many negative vertices. Let $\mathcal{F}^{*}$ be the modified surface with no $a b$-tiles. Suppose the tiling of $\mathcal{F}^{*}$ contains a $b b$-tile. This $b b$-tile must be contained in a component of $\mathcal{F}^{*}$ tiled entirely by $b b$-tiles, since any connected component containing both $a a$-tiles and $b b$-tiles must contain at least one $a b$-tile. But then $\mathcal{F}^{*}$ contains a closed component, a contradiction. Therefore, $\mathcal{F}^{*}$ is tiled entirely by $a a$-tiles, i.e. $\mathcal{F}^{*}$ is a special Markov surface. $\|$

Corollary to Theorem 4.2 (Markov's Theorem for the case of the unknot) $[\mathbf{B e}]$. Let $K$ be any closed braid representative of the unknot. Let $U$ be the standard 1-braid representative. Then there exists a finite sequence of closed braid representatives $K=K_{0} \rightarrow K_{1} \rightarrow \ldots K_{r}=U$ and $1 \leq p \leq r$ such that if $i \leq p$ (resp. $i>p$ ) then $K_{i}$ is obtained from $K_{i-1}$ by a single stabilization along an ab tile (resp. destabilization via a type (a) vertex. 
Proof of the Corollary: Since $K$ is the unknot, it is the boundary of a Markov surface $\mathcal{F}=\mathcal{F}_{1}$, which is a topological disc. By Theorem 2.2 we may stabilize along $a b$ tiles to obtain from $\mathcal{F}_{\text {, a special Markov surface which we call } \mathcal{F}}$. Its boundary is $K_{p}$, and the moves from $K_{0}$ to $K_{p}$ are as claimed. This new surface is also a disc, and it is tiled entirely by $a a$ tiles. In the new tiling the graphs $G_{+,+}$and $G_{+,-}$must both be trees, in view of Theorem 3.1 and the fact that $\mathcal{F}_{\sqrt{ }}$ is a disc. Each endpoint vertex is then a vertex of type $(a)$. Destabilizing, we reduce the number of vertices but leave the tiling unaltered outside the tile we just removed. In this way the endpoint vertices may be removed, one after another, until we obtain a special Markov surface $\mathcal{F}_{\nabla}$ which is radially foliated. Its boundary $K_{r}$ is the standard 1braid representative $U$ of the unknot. $\|$

Problem 4.3. A special Markov surface is said to be a Bennequin surface if its boundary has minimal braid index. In the manuscript BM 3] it is proved that every link which is represented as a closed n-braid with $n \leq 3$ is the boundary of a Bennequin surface. We ask: what are necessary and sufficient conditions for a link to bound a Bennequin surface.

Our final application is to give a simple proof of the main result from BM 5], i.e. that there is a systematic procedure for simplifying an arbitrary closed braid representative of the $r$-component unlink to the trivial closed $r$ braid. The simple proof uses the results in $\S 3$, which were not available when this result was first proved. After we complete the proof we will illustrate its meaning via an example:

Theorem 4.3 (Markov's theorem without stabilization in the case of the unlink) [BM 5]. Every closed braid representative $L$ of the $r$ component unlink $\mathcal{U}_{r}$ may be reduced to the standard $r$-braid representative $U_{r}$ by a finite sequence of the following moves: exchange moves, isotopy of $L$ in the complement of the braid axis, and destabilizations along vertices of type (a).

Proof of Theorem 4.3. Choose an arbitrary closed braid representative $L$ of $\mathcal{U}_{r}$. Our closed braid $L$ is the boundary of a Markov surface $\mathcal{F}$ which is a union of $r$ disjoint discs $D_{1}, \ldots, D_{r}$, each of which admits a tiling. We will show that, after some number of changes in foliation, exchange moves, isotopies in the complement of the axis and destabilizations along vertices of type $(a)$, each $D_{i}$ is foliated entirely by $a$-arcs.

By Theorem 3.1 (iii), we may assume that no closed loop in $G_{\epsilon, \delta}$ bounds 
a disk on any disk $D_{i}$. Since every closed loop on a disc bounds a disc, we conclude that each component of $G_{\epsilon, \delta}$ is a tree or an isolated vertex. By Theorem 3.1 (ii), each component of $G_{-,-}$is a tree. Suppose there is such a tree component $T$. Let $\alpha$ be an edgepath of $T$ connecting two endpoints of $T$. By Theorem 3.1 (i), $\partial \alpha \subset L$. But then $\alpha$ separates some $D_{i}$ into two subdisks, each of which is bounded by loop consisting of edges in $G_{-,-}$and a subarc of $L$. Since this contradicts Theorem 3.1 (v), we may assume that $G_{-,-}=\emptyset$.

Suppose there is a tree component $T$ of $G_{+,+}$. If $v$ is an endpoint vertex of $T$, then $\operatorname{star}(v)$ contains exactly one positive vertex. Since $G_{-,-}=\emptyset$, there are no negative singularities in $\operatorname{star}(v)$. Thus, $v$ has valence 1 , and it may be eliminated by a destabilization move. Induction on the number of endpoints of $T$, allows us to conclude that $G_{+,+}$consists entirely of isolated vertices. But then there are no singularities in the foliation of $\mathcal{F}$ and, consequently, each $D_{i}$ is foliated entirely by $a$-arcs. $\|$

Example 4.1. We illustrate Theorem 4.3 with an example. The example, which is indicated in Figures 4.1, suggests an infinite sequence of closed 4braids, all of which determine the same knot type. We examine this example in the special case of the unknot which occurs when $X=\sigma_{2}$ and $Y=\sigma_{2} \sigma_{1}$.

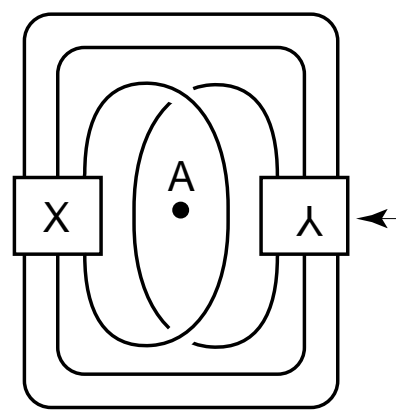

(a)

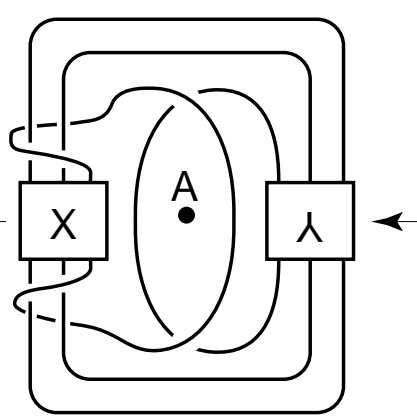

(b)

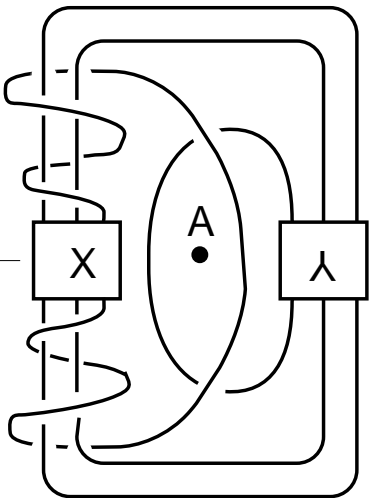

(c)

Figure 4.1

Thomas Fiedler [Fie has introduced an invariant of conjugacy class and used it to prove that when $X=\sigma_{1} \sigma_{2}$ and $Y=\sigma_{2}$ these braids are in infinitely many distinct conjugacy classes, that is, no two closed braids in the sequence are isotopic in the complement of the braid axis $A$. We will see in our case, which is similar, that the foliation of the disk bounded by the unknot indicates that any two adjacent braids in the sequence are related by 
an exchange move. (Remark: since exchange moves reduce complexity, the arrows $4.1(c) \rightarrow 4.1(b) \rightarrow 4.1(a)$ are in the correct direction). The foliation of the disk bounded by the unknot in Figure 4.1(a) will then show that the standard representative of the unknot can be obtained after destabilizations along vertices of type $(a)$. Thus, this example illustrates how a very complicated braid representative for the unknot may be transformed into the standard one by a sequence of the moves described in Theorem 4.3.

To begin to understand this example in terms of the foliated disks which the knot bounds, the reader should first verify that the knot projections shown in Figures 4.1(c),(b),(a) correspond to the knots embedded in 3-space of Figures 4.2(c),(b),(a), respectively. The tilings of the disks bounded by these knots are shown in Figures 4.3(c),(b),(a), respectively. To see that the embedded surfaces in Figures 4.2 are in fact discs and that the tilings in Figures 4.3 are the correct ones for the disks in Figures 4.2, it is helpful to first study Figures 4.2(a), 4.3(a) and then the transition from Figures 4.2(a), 4.3(a) to Figures 4.2(b), 4.3(b), respectively.

The reader should have no trouble verifying that surface in Figure 4.2(a) is a disk and that the singular leaves of its tiling are as shown in Figure 4.3(a). Note that, running along the boundary of the closed braid in Figures $4.2(\mathrm{a})$ and $4.3(\mathrm{a})$, starting at the point $q_{+}$, one encounters the points $q_{+}, q_{-}, r_{-}, r_{+}, p_{+}, p_{-}$in that order. These points are the endpoints on the unknot of subarcs $p_{+} p_{-}, q_{+} q_{-}$, and $r_{+} r_{-}$of the three singular leaves in the tiling shown in 4.3(a). The points where the axis pierces the disc in $4.2(\mathrm{a})$ are numbered $1,2,3,4$. The singular leaf $p_{+} p_{-}$cuts off a subdisc $P$ which contains 1 , and similarly there are subdiscs $Q, R$ containing 3,4 which are cut off by $q_{+} q_{-}, r_{+} r_{-}$. The center subdisc, containing 2 , will be called $W$. From Figure 4.2(a) we see that the subdiscs $P, W, R, Q$ are pierced by $A$ in that order. Note that the surface in Figure 4.2(b) can be obtained from the one in Figure 4.1(a) by first creating "pockets" in $P$ and $W$ (which add new vertices $u_{1}, u_{2}, v_{1}, v_{2}$ to the tiling) and then lifting the disk $Q$ up and around and under the braid $X$ and then pushing it back up onto the axis again by slipping it into the pockets in $P$ and $W$. Thus, the surface in Figure 4.2(b) is a disk. To see that its tiling is as shown in Figure 4.3(b), note that each pocket is formed by the union of two $a b$-tiles joined at their bounding $a$-arcs and $b$-arcs. The singular leaves of the $a b$-tiles must be as shown in Figure 4.3(b), due to the cyclic order in the fibration of the singularities in the tiling of Figure 4.2(b).

Similarly, the embedding shown in Figure 4.2(c) can be obtained from the embedding in Figure 4.2(b) by creating new pockets in $P$ and $W$ and slipping the old pockets together with $Q$ around $X$ and up into the new pockets through the axis. Again, the tiling corresponding to Figure 4.2(c) 
must be as shown in Figure 4.3(c), due to the cyclic order in the fibration of the singularities in the tiling of Figure 4.2(c). Continuing in this manner, 


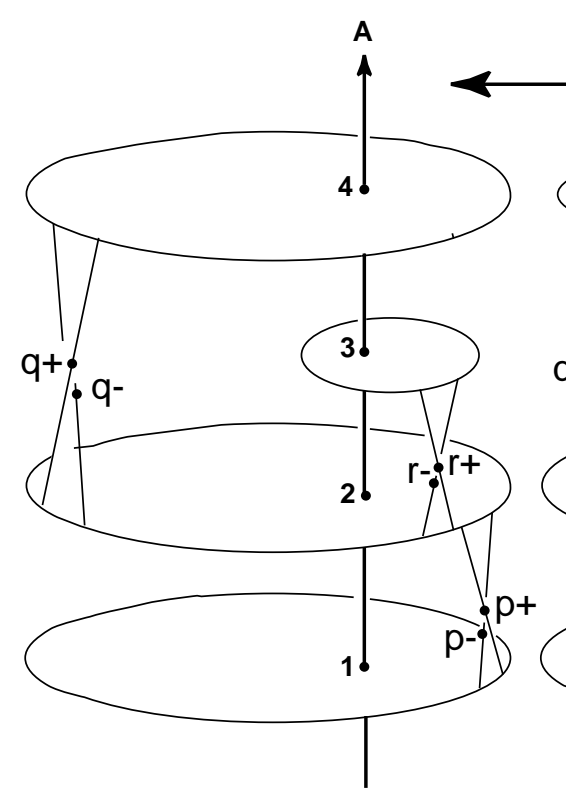

(a)

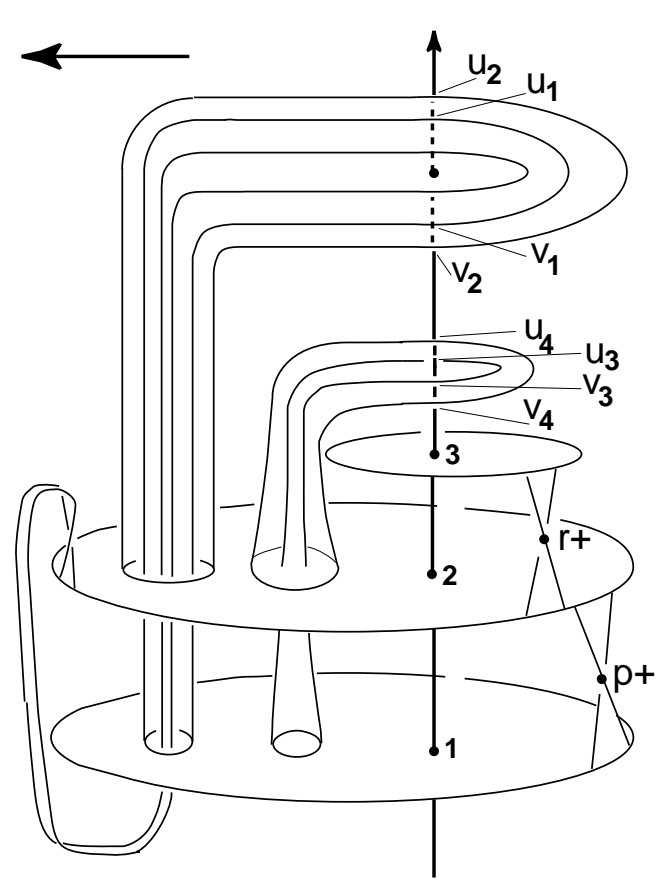

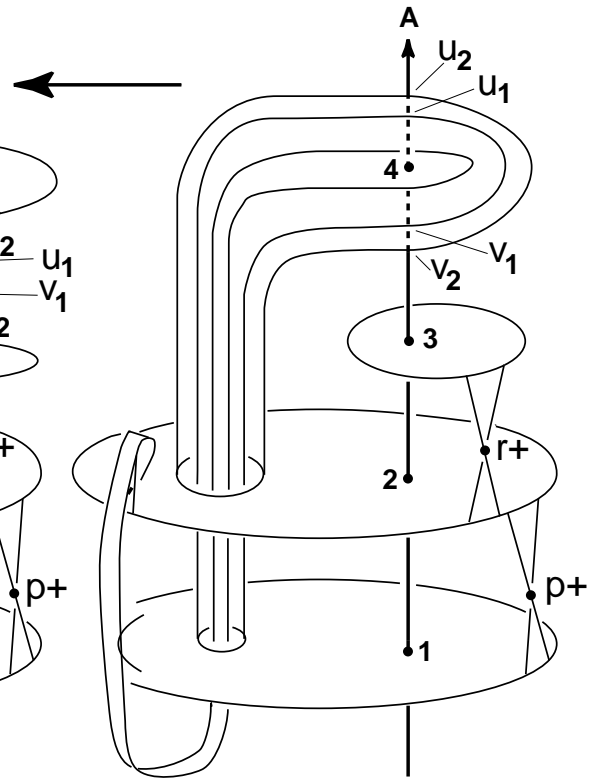

(b)

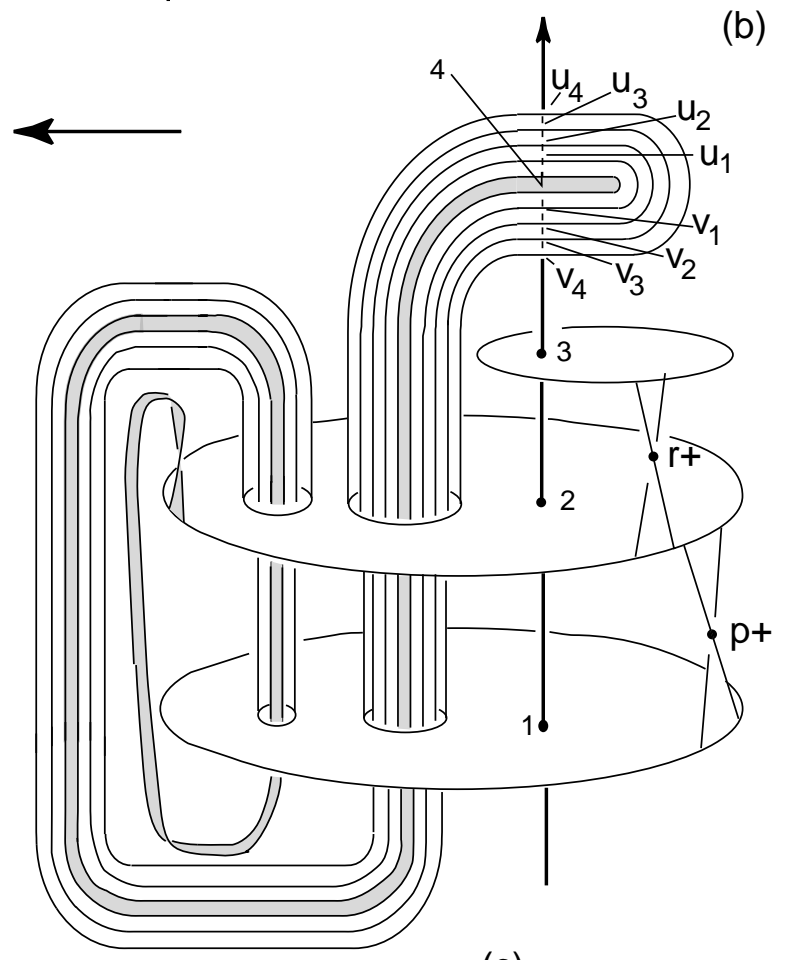

(c)

Figure 4.2 
we may obtain embeddings and tilings for all disks bounded by our infinite sequence of unknots.

To see that the passages from Figures (c) to (b) to (a) illustrate the moves of Theorem 4.3, note that each of the vertices $v_{1}, v_{2}, v_{3}, v_{4}$ in Figure $4.3(\mathrm{~b})(\mathrm{c})$ has valence 2 , type $(b, b)$ and sign $(+,-)$. Thus, by Theorem 2.2 , each such vertex determines an exchange move. The passage from Figure $4.3(\mathrm{c})$ to $(\mathrm{b})$ is realized by the two exchange moves which are determined by the vertices $v_{3}, v_{4}$, and the passage from Figure 4.3(b) to (a) is realized by the two exchange moves which are determined by the vertices $v_{1}, v_{2}$. Thus, the moves which take us from Figures $4.2(\mathrm{c})$ to $4.2(\mathrm{~b})$ and $4.2(\mathrm{~b})$ to $4.2(\mathrm{a})$ are in each case two exchange moves, each of which empties a pocket and is followed by deletion of inessential $b$-arcs, i.e. by the collapse of the pocket. In this manner, we can pass from any knot in our infinite sequence down to Figure 4.2(a) via exchange moves. Now note that the vertices $1,3,4$ in Figure 4.3(a) all have type $(a)$. Thus, after destabilizing along each of these vertices, we obtain the standard representative of the unknot from the disk in Figure 4.2(a).

Remark 4.2 Example 4.1 does not really illustrate the full power of Theorem 4.3. There are vertices of type $(a)$ in the foliated discs of Figures 4.3(c),(b),(a) and so our foliated discs can be simplified to yield 3-braid examples via destabilization. Since all closed 3 and 2-braid representatives of the unknot can be reduced to the trivial 1-braid representative without using exchange moves [BM 3] the need for exchange moves will thus disappear.

There is another closed 4-braid representative of the knot, due to Hugh Morton (see [BM 50), which is more complicated than the one we have shown because the associated foliated disc does not contain a vertex of type $(a)$. By Theorem 4.3 the only way one can simplify that foliation without increasing braid index is by first using (two) exchange moves. Unfortunately, however, that example was simply too complicated for us to be able to illustrate it (and the pictures in Figure 4.2 should make it clear why)!

Our examples do, nevertheless, illustrate a related point. If one goes back to Figure 4.1 and chooses arbitrary 3-braids $X$ and $Y$ in place of the braids $\sigma_{2}$ and $\sigma_{2} \sigma_{1}$ which were illustrated in Figures 4.2 and 4.3 , then one may easily obtain a more complicated knot $K$ which bounds a surface $\mathcal{F}$ (now of genus $>0$ ), and the foliation of that surface will illustrate the need for exchange moves because there will not be any vertices of type $(a)$. On the other hand, foliated surfaces of genus $>0$ present their own difficulties when one tries to illustrate them, and so we settled for an example which illustrated the phenomenon in a case where it was possible to make it concrete. 


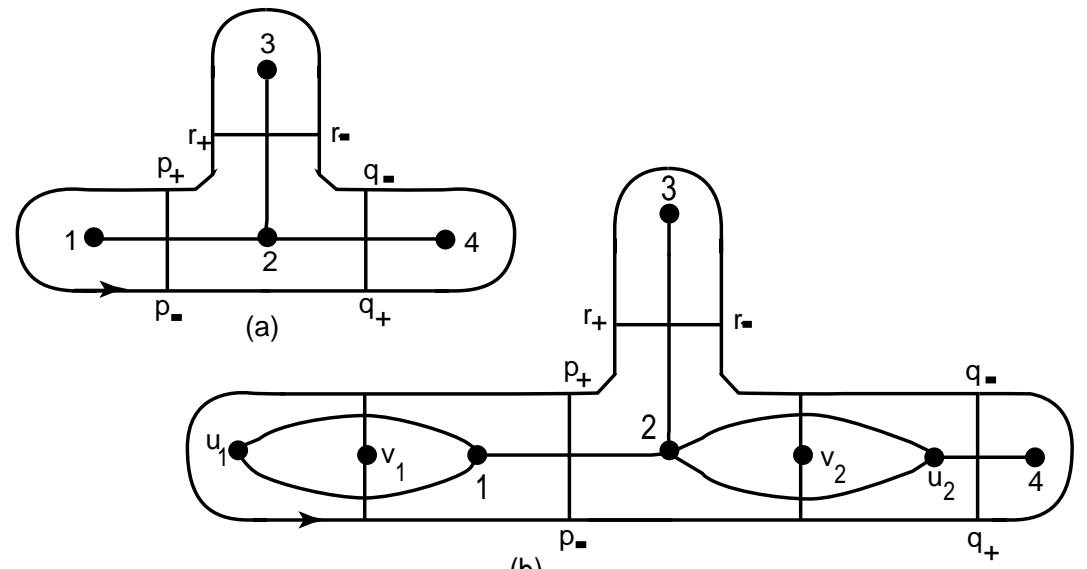

(b)

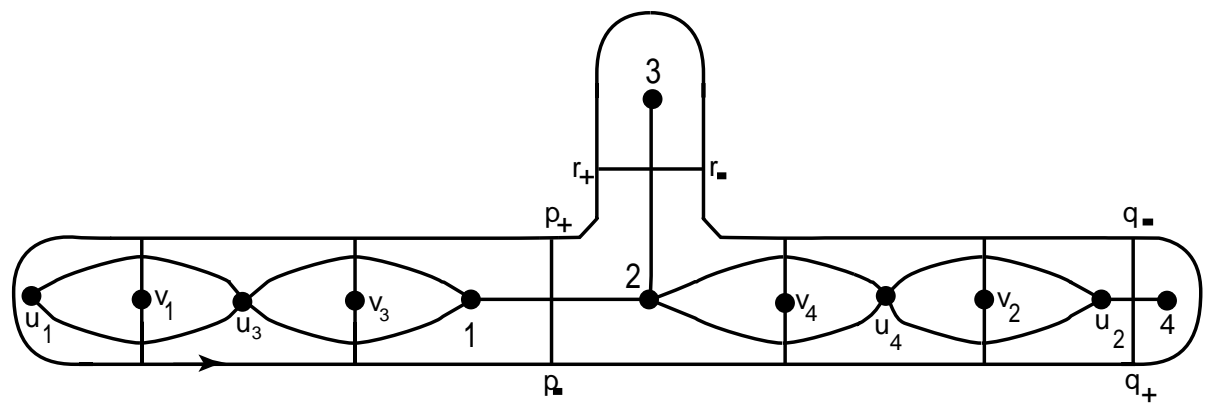

(c)

Figure 4.3

Remark 4.3. Theorem 4.3 is a much stronger result than the Corollary to Theorem 4.2, or Markov's well-known theorem for arbitrary knots and links, because the moves which are used in Theorem 4.3 either preserve or reduce braid index. Thus Theorem 4.3 is "Markov's theorem without stabilization", in the special case of the unlink. On the other hand, Theorem 4.2 illustrates the powerful role which stabilization plays, enabling us, as it does, to remove all the 'bubbles' from an arbitrary Markov surface and replace it with one which has minimal genus and only discs which are joined by unknotted halftwisted bands. Unfortunately, however, these 'nice' Markov surfaces are not, in general, bounded by closed braids which have minimal braid index for the given knot type.

In the case of more general knots and links, Markov's theorem without stabilization requires braid preserving flypes in addition to the exchange moves of Theorem 4.3. A rudimentary version of the braid-preserving flype 
plays an important role in the work in [BM 3], which is Markov's theorem without stabilization in the special case of links which are closed 3-braids.

Problem 4.4 In the manuscript [ [] J. Los has applied ideas from [BM 7] to generalize Theorem 4.3 to the case of braided satellite knots. He has proved that by repeated use of isotopy, exchange moves and destabilization along vertices of type $(a)$ any closed braid representative of a braided satellite knot can be reduced to minimum braid index. Such a result is false for arbitrary closed braid representatives of arbitrary links, nevertheless there should be very general cases under which it is true. We pose this as an open problem.

Problem 4.5 In the same manuscript [ [ ] J. Los has proved that torus knots (like the unknot and unlink) admit a unique closed braid representative of minimum genus. Examples are given in BM 3] and general mechanisms are constructed in [BM 6] which show that this is not true in general. We pose the open problem of finding other cases when it is true.

Problem 4.6 How can one generalize the graphs $G_{\epsilon, \delta}$ to related graphs on foliated closed embedded surfaces? Do they simplify the results in [BM 7], [Fin, BM 4]? 


\section{References}

[A] A.M. Akimenkov, "Solution of the reducibility problem for 4-string braids", Int. J. of Algebra and Computation 1, No. 2 (1991), 185-200.

[Al] J.W. Alexander, "A lemma on systems of knotted curves", Proc. Nat. Acad. Sci., U.S.A. 9 (1923), 93-95.

[Be] D.Bennequin, "Entrelacements et equations de Pfaff", These de Doctorat d'Etat, Universite de Paris VII, 24 novembre 1982. English version: Russian Math.Surveys 44, No. 3 (1989), 1-65.

[BM 1] J.Birman and W.Menasco, "Studying links via closed braids I: A finiteness theorem", Pacific Journal of Mathematics 154, No. 1 (1992), 17-36.

[BM 2] J.Birman and W.Menasco, "Studying links via closed braids II: On a theorem of Bennequin", Topology and its Applications 40 (1991), 71-82.

[BM 3] J.Birman and W.Menasco, "Studying links via closed braids III: Classifying links which are closed 3-braids", Pacific Journal of Mathematics 161, No. 1 (1993), 25-113.

[BM 4] J.Birman and W.Menasco, "Studying links via closed braids IV: Split links and composite links", Inventiones mathematicae 102 (1990), 115-139.

[BM 5] J.Birman and W.Menasco, "Studying links via closed braids V: The unlink", Trans. AMS 329, No. 2 (1992), 585-606.

[BM 6] J.Birman and W.Menasco, "Studying links via closed braids VI: A non-finiteness theorem", Pacific Journal of Mathematics 156, No. 2 (1992), 265-285.

[BM 7] J.Birman and W.Menasco, "Special positions for essential tori in link complements", Topology 33, No. 3 (1994), 525-556. 
[C] P. Cromwell, "Embedding knots and links in open books I", Topology and its applications 64, No. 1 (1995), 37-58.

[Fie] T. Fiedler, "A small state sum for knots", Topology 32, No. 2 (1993), 281-294.

[Fin] E. Finkelstein, "Closed incompressible surfaces in closed braid complements", The Journal of Knot Theory and its Ramifications, same issue as this paper.

[Fu] T.S. Fung, "On clasp and ribbon surfaces bounded by a closed braid", PhD Thesis, Columbia University, 1996.

[KL] K.Y. Ko and S.J. Lee "Genera of some closed 4-braids", preprint, 1995.

[L] J.E.Los, "Knots, braid index and dynamical type", Topology 33, No. 2 (1994), 257-270.

[M] W.W.Menasco, "The Bennequin-Milnor unknotting conjecture", C.R. Acad. Sc. Paris, t.318, Serie I (1994), 831-836.

[N] T. Nowik, "Intersection of surfaces in 3-manifolds", PhD Thesis, Hebrew University, 1996. 